\title{
Green's canonical syzygy conjecture for generic curves of odd genus
}

\author{
Claire Voisin
}

\begin{abstract}
We prove in this paper the Green conjecture for generic curves of odd genus. That is, we prove the vanishing $K_{k, 1}\left(X, K_{X}\right)=0$ for $X$ a generic curve of genus $2 k+1$. This completes our previous work, where the Green conjecture for generic curves of genus $g$ with fixed gonality $d$ was proved in the range $d \geqslant g / 3$, with the possible exception of the generic curves of odd genus. The case of generic curves of odd genus was considered as especially important, since Hirschowitz and Ramanan proved that if the conjecture is true for the generic curve of odd genus, then the locus of jumping syzygies is exactly the locus of exceptional gonality, as predicted by Green's conjecture. Thus, our result combined with the Hirschowitz-Ramanan result is a strong confirmation of Green's conjecture.
\end{abstract}

\section{Introduction}

For $X$ a projective variety, $L$ a line bundle on $X$, and $\mathcal{F}$ a coherent sheaf on $X$, denote by $K_{p, q}(X, L, \mathcal{F})$, following [Gre84], the cohomology at the middle of the exact sequence

$$
\begin{aligned}
\bigwedge^{p+1} H^{0}(X, L) \otimes H^{0}(X, \mathcal{F}((q-1) L)) & \rightarrow \bigwedge^{p} H^{0}(X, L) \otimes H^{0}(X, \mathcal{F}(q L)) \\
& \rightarrow \bigwedge^{p-1} H^{0}(X, L) \otimes H^{0}(X, \mathcal{F}((q+1) L)),
\end{aligned}
$$

where the maps are Koszul differentials. For $\mathcal{F}=\mathcal{O}_{X}$, use the notation $K_{p, q}(X, L)$. Green's conjecture on syzygies of canonical curves (see [Gre84]) relates the Koszul cohomology groups

$$
K_{p, 1}\left(C, K_{C}\right),
$$

for $C$ a smooth projective curve, to the Clifford index of the curve,

$$
\operatorname{Cliff}(C):=\operatorname{Min}_{D}\{\operatorname{deg} D-2 r\},
$$

where $D$ runs through the set of divisors $D$ on $C$ satisfying

$$
r+1:=h^{0}(D) \geqslant 2, h^{1}(D) \geqslant 2 .
$$

Conjecture 1 (Green).

$$
K_{l, 1}\left(C, K_{C}\right)=0, \quad \forall l \geqslant p \Leftrightarrow \operatorname{Cliff}(C)>g-p-2 .
$$

The direction $\Rightarrow$ is proved by Green and Lazarsfeld in the appendix to [Gre84]. The case $p=g-2$ of the conjecture is equivalent to Noether's theorem, and the case $p=g-3$ to Petri's theorem (see [GL84]). The case $p=g-4$ has been proved in any genus by Schreyer [Sch91] and by the

Received 22 January 2003, accepted in final form 21 October 2004, published online 1 September 2005. 2000 Mathematics Subject Classification 14H99, $14 \mathrm{~J} 28$.

Keywords: canonical curves, canonical syzygies, Green's conjecture, K3 surfaces.

This journal is (C) Foundation Compositio Mathematica 2005. 


\section{VoIsin}

author [Voi88] for $g>10$. The vanishing of $K_{l, 1}\left(C, K_{C}\right)$ for $l$ large with respect to $g$ was proved by Ein [Ein87].

In [GL87], Green and Lazarsfield provided beautiful evidence for the conjecture, by proving that the Clifford index is constant for curves in linear systems on a K3 surface. More recently, the conjecture has been studied in [Tei02, Voi02], for generic curves of fixed gonality. Teixidor proves the following theorem.

Theorem 1 [Tei02]. Green's conjecture is true for generic curves of genus $g$ and fixed gonality $\gamma$, in the range

$$
\gamma \leqslant \frac{g+7}{3}
$$

Note that Brill-Noether theory says that the gonality $\gamma$ always satisfies the inequality

$$
\gamma \leqslant\left[\frac{g+3}{2}\right]
$$

with equality for the generic curve. We have proved the following theorem.

Theorem 2 [Voi02]. Green's conjecture is true for generic curves of genus $g$ and fixed gonality $\gamma$, in the range

$$
\gamma \geqslant \frac{g}{3}
$$

except possibly for the generic curves of odd genus $g=2 k+1$, whose gonality is $(k+2)$.

So, for generic curves of fixed gonality, the only remaining case is that of generic curves of odd genus $g=2 k+1$. Green's conjecture together with Brill-Noether theory predicts that

$$
K_{k, 1}\left(C, K_{C}\right)=0 .
$$

This is the main result proved in this paper. We give the precise statement below; it gives slightly more, since it proves the vanishing (1) for some explicit curves which we know to be generic in the Brill-Noether sense. Applications of this result to the gonality conjecture for generic curves can be found in [AV03, Apr04].

Note that this last case was especially challenging: firstly, because, as noticed in [HR98], the locus of jumping syzygies, i.e. the locus where $K_{k, 1}\left(C, K_{C}\right) \neq 0$, is of codimension 1 in $\mathcal{M}_{g}$ in this case, and in fact has a natural structure of determinantal hypersurface; and secondly because of the following important result of Hirschowitz and Ramanan.

Theorem 3 [HR98]. If the Green conjecture is true for generic curves of genus $(2 k+1)$, then the locus of jumping syzygies in $\mathcal{M}_{2 k+1}$ is equal set theoretically to the $(k+1)$-gonal divisor, which is also the locus where the Clifford index is one less the generic Clifford index.

Combined with the generic Green conjecture for genus $(2 k+1)$-curves, this provides strong evidence for Conjecture 1. In [Apr05], Aprodu subsequently proved that the Green conjecture is satisfied by curves satisfying a certain Brill-Noether property, using our results and the argument of Hirschowitz and Ramanan extended to nodal curves.

Coming back to our result, the curves we consider are the following: we consider a smooth projective K3 surface $S$, such that $\operatorname{Pic}(S)$ is isomorphic to $\mathbb{Z}^{2}$, and is freely generated by $L$ and $\mathcal{O}_{S}(\Delta)$, where $\Delta$ is a smooth rational curve such that $\operatorname{deg} L_{\mid \Delta}=2$, and $L$ is a very ample line bundle with $L^{2}=2 g-2, g=2 k+1$. By the hyperplane section theorem (see [Gre84]), we have

$$
K_{k, 1}(S, L) \cong K_{k, 1}(C, L), \quad \forall C \in|L| \text {. }
$$




\section{GREEN'S CANONICAL SYZYGY CONJECTURE FOR GENERIC CURVES OF ODD GENUS}

As we shall see in the next section, curves in $|L|$ have the generic Clifford index. Hence we expect from Green's conjecture that

$$
K_{k, 1}(C, L)=0=K_{k, 1}(S, L) .
$$

Our theorem does indeed say the following.

TheOREM 4. The K3 surface $S$ being as above, we have

$$
K_{k, 1}(S, L)=0 \text {. }
$$

In the first section, we show how to adapt the arguments of [Voi02] to the line bundle $L+\Delta$ on $S$, in order to show that

$$
K_{k+1,1}(S, L+\Delta)=0
$$

Note that the proof of [Voi02] worked under the assumption that $\operatorname{Pic}(S)=\mathbb{Z}$, which is why a few supplementary arguments are needed.

In the second section, we show how to deduce from this the vanishing $K_{k, 1}(S, L)=0$. The last section is devoted to the proof of the crucial Proposition 8 used in the proof of Theorem 4 .

\section{The case of curves of even genus on a K3 surface with a node}

Let $S$ be a K3 surface, whose Picard group is freely generated by a very ample line bundle $L$, such that

$$
L^{2}=2 g-2, \quad g=2 k+1,
$$

and $\mathcal{O}_{S}(\Delta)$, where $\Delta$ is a rational curve such that

$$
\operatorname{deg} L_{\mid \Delta}=2 .
$$

Let $L^{\prime}=L(\Delta)$; smooth curves in $\left|L^{\prime}\right|$ do not meet $\Delta$ and are of genus $2 k+2=2(k+1)$. Contracting $\Delta$ to a node, the line bundle $L^{\prime}$ descends, and we are essentially in the situation considered in [Voi02]. (Note, however, the change of notation from $k$ to $k+1$.)

We first apply Lazarsfeld's argument in [Laz86] to show the following proposition.

Proposition 1. Smooth curves $C$ in $|L|$ or in $\left|L^{\prime}\right|$ are generic in the Brill-Noether sense, i.e. they do not have a $g_{d}^{r}$ when the Brill-Noether number $\rho(g(C), d, r)$ is negative. In particular, their Clifford index is the generic one.

Proof. It follows from [Laz86] that if $C \subset S$ is a smooth curve in a linear system $|M|$, and $D$ is a $g_{d}^{r}$ on $C$ with $\rho(g(C), d, r)<0$, then there exists a line bundle $H$ on $S$ with

$$
h^{0}(H) \geqslant 2, \quad h^{0}(M-H) \geqslant 2 .
$$

Apply this to $M=L$ or $M=L^{\prime}$. Writing $H=\alpha L+\beta \Delta$, the condition $h^{0}(H) \geqslant 2$ implies that $\alpha \geqslant 1$. Similarly, the condition $h^{0}(M-H) \geqslant 2$ implies that $1-\alpha \geqslant 1$, whether $M=L$ or $M=L^{\prime}$. This is a contradiction.

It is now expected from Green's Conjecture 1 that

$$
K_{k+1,1}\left(S, L^{\prime}\right)=0, \quad K_{k, 1}(S, L)=0 .
$$

In [Voi02], we proved the vanishing

for a line bundle $L^{\prime}$ on $S$ with

$$
K_{k+1,1}\left(S, L^{\prime}\right)=0
$$

$$
L^{\prime 2}=2 g^{\prime}-2, \quad g^{\prime}=2 k+2,
$$

under the assumption that $L^{\prime}$ generates $\operatorname{Pic}(S)$. Our first goal is to extend this result in our situation. 


\section{VoIsin}

Theorem 5. For $S, L^{\prime}=L(\Delta)$ as above, we have

$$
K_{k+1,1}\left(S, L^{\prime}\right)=0 .
$$

The proof of this theorem occupies the rest of this section. Let $C^{\prime} \in\left|L^{\prime}\right|$ be smooth; by BrillNoether theory, there is a smooth $g_{k+2}^{1}$, say $D$, on $C^{\prime}$. By Proposition 1 , both $D$ and $K_{C^{\prime}}-D$ are generated by sections. Consider the Lazarsfeld bundle

$$
E=F^{*}=F \otimes L^{\prime},
$$

where $F$ is the rank 2 vector bundle fitting in the exact sequence

$$
0 \rightarrow F \rightarrow H^{0}\left(C^{\prime}, D\right) \otimes \mathcal{O}_{S} \rightarrow D \rightarrow 0 .
$$

Here the last map is the evaluation map along $C^{\prime}$. One can show that $E$ does not depend on the curve $C^{\prime}$, or on the curve $D^{\prime}$. The bundle $E$ has $\operatorname{det} E \cong L^{\prime}$, and $h^{0}(E)=k+3$. The following key point, which was used constantly throughout the proof of [Voi02], remains true in our situation.

Proposition 2. The determinant map

$$
d: \bigwedge^{2} H^{0}(S, E) \rightarrow H^{0}\left(S, L^{\prime}\right)
$$

does not vanish on decomposable elements.

Proof. Indeed, assume $s, s^{\prime} \in H^{0}(S, E)$ are not proportional but satisfy $d\left(s \wedge s^{\prime}\right)=0$. Then $s, s^{\prime}$ generate a sub-line bundle of $E$, say $H$, which we may assume to be saturated, and which satisfies

$$
h^{0}(H) \geqslant 2 \text {. }
$$

Hence there is an exact sequence

$$
0 \rightarrow H \rightarrow E \rightarrow H^{\prime} \rightarrow T \rightarrow 0
$$

where $H^{\prime}$ is a line bundle such that $H+H^{\prime}=\operatorname{det} E=L^{\prime}$, and $T$ is torsion supported on points of $S$. Since $E$ is generated by sections, $H^{\prime}$ is generated by sections away from the support of $T$. On the other hand, $H^{\prime}$ is not trivial, since $H^{0}\left(S, E^{*}\right)=0$. So $h^{0}\left(H^{\prime}\right) \geqslant 2$. But this contradicts the fact we already mentioned, that we cannot write $L^{\prime}$ as the sum of two line bundles admitting at least two sections.

We now recall the main points of the proof of the vanishing (2) given in [Voi02], in order to make clear what has to be added in our situation. We warn the reader again that the notation of [Voi02] has been shifted (the integer $k$ there becomes $(k+1)$ here).

First step. Let $S_{\text {curv }}^{[k+2]}$ be the open subset of the Hilbert scheme of $S$ parametrizing curvilinear, degree $k+2$, zero-dimensional subschemes of $S$. Let

$$
I_{k+2} \stackrel{\pi_{k+2}}{\longrightarrow} S_{\text {curv }}^{[k+2]}, I_{k+2} \subset S \times S_{\text {curv }}^{[k+2]}
$$

be the incidence scheme. We established the following isomorphism:

$$
K_{k+1,1}\left(S, L^{\prime}\right) \cong H^{0}\left(I_{k+2}, \pi_{k+2}^{*} L_{k+2}^{\prime}\right) / \pi_{k+2}^{*} H^{0}\left(S_{\text {curv }}^{[k+2]}, L_{k+2}^{\prime}\right),
$$

where the line bundle $L_{k+2}^{\prime}$ is the determinant of the vector bundle $\mathcal{E}_{L^{\prime}}$ of rank $k+2$ on $S_{\text {curv }}^{[k+2]}$, defined as

$$
\mathcal{E}_{L^{\prime}}=R^{0} \pi_{k+2 *}\left(p r_{1}^{*} L^{\prime}\right)
$$

From this we deduced the following criterion. 


\section{GREEN'S CANONICAL SYZYGY CONJECTURE FOR GENERIC CURVES OF ODD GENUS}

Lemma 1. The vanishing $K_{k+1,1}\left(S, L^{\prime}\right)=0$ holds if there exist a reduced scheme $Z$ and a morphism

$$
j: Z \rightarrow S_{\text {curv }}^{[k+2]}
$$

such that, denoting

$$
\widetilde{Z} \stackrel{\tilde{j}}{\rightarrow} I_{k+2}
$$

the fibered product

$$
\widetilde{Z}=Z \times_{S_{\text {curv }}^{[k+2]}} I_{k+2},
$$

we have:

(i) the map

$$
\tilde{j}^{*}: H^{0}\left(I_{k+2}, \pi_{k+2}^{*} L_{k+2}^{\prime}\right) \rightarrow H^{0}\left(\widetilde{Z}, \tilde{j}^{*} \pi_{k+2}^{*} L_{k+2}^{\prime}\right)
$$

is injective;

(ii) denoting by $\pi: \widetilde{Z} \rightarrow Z$ the first projection, the map

$$
\pi^{*}: H^{0}\left(Z, j^{*} L_{k+2}^{\prime}\right) \rightarrow H^{0}\left(\widetilde{Z}, \tilde{j}^{*} \pi_{k+2}^{*} L_{k+2}^{\prime}\right)
$$

is surjective.

Second step. The construction of $Z$ is as follows: we start with the vector bundle $E$ of (3) and (4). It has $c_{2}(E)=k+2$. Denote by

$$
\mathbb{P}\left(H^{0}(E)\right)_{\text {curv }} \subset \mathbb{P}\left(H^{0}(S, E)\right)
$$

the open set parametrizing sections $\sigma \in H^{0}(S, E)$ whose zero-scheme $z_{\sigma}$ is zero-dimensional and curvilinear. There is a natural morphism

$$
\begin{gathered}
\mathbb{P}\left(H^{0}(E)\right)_{\text {curv }} \rightarrow S_{\text {curv }}^{[k+2]} \\
\sigma \mapsto z_{\sigma} .
\end{gathered}
$$

Let $W=\mathbb{P}\left(H^{0}(E)\right)_{\text {curv }} \times_{S_{\text {curv }}^{[k+2]}} I_{k+2}$. This is a cover of $\mathbb{P}\left(H^{0}(E)\right)_{\text {curv }}$ of degree $k+2$. It admits a natural morphism, say $f$ to $I_{k+2}$. We use now the morphism

$$
\tau_{k+2}: I_{k+2} \rightarrow S_{\text {curv }}^{[k+1]},
$$

which sends a point $(x, z),\{x\} \subset z$ of $I_{k+2}$ to the residual scheme of $x$ in $z$, which is curvilinear of length $k+1$, since $z$ is curvilinear. Let

$$
\psi: W \rightarrow S_{\text {curv }}^{[k+1]}
$$

be the composed map $\psi=\tau_{k+2} \circ f$. Finally we construct the sum map

$$
\begin{gathered}
j: Z:=(\widetilde{S \times W})_{0} \rightarrow S_{\text {curv }}^{[k+2]}, \\
(x, w) \mapsto x \cup \psi(w),
\end{gathered}
$$

where the here means to 'blow-up along the incidence subscheme in order to make the scheme structure on the union $x \cup \psi(w)$ well defined', and the subscript 0 means 'taking an open set in order to make sure that this scheme structure is curvilinear'.

Third step. The injectivity of the map (6) in Lemma 1 is easily reduced to the injectivity of the restriction map

$$
\psi^{*}: H^{0}\left(S_{\text {curv }}^{[k+1]}, L_{k+1}^{\prime}\right) \cong \bigwedge^{k+1} H^{0}\left(S, L^{\prime}\right) \rightarrow H^{0}\left(W, \psi^{*} L_{k+1}^{\prime}\right) .
$$




\section{VoIsin}

Now if $\beta: W \rightarrow \mathbb{P}\left(H^{0}(E)\right)_{\text {curv }}$ is the natural surjective map, we showed that

$$
\psi^{*} L_{k+1}^{\prime} \cong \beta^{*} \mathcal{O}_{\mathbb{P}\left(H^{0}(E)\right)_{\text {curv }}}(k+1)
$$

and that the map above is the composition of $\beta^{*}$ and of an isomorphism

$$
\bigwedge^{k+1} H^{0}\left(S, L^{\prime}\right) \cong H^{0}\left(\mathbb{P}\left(H^{0}(E)\right)_{\mathrm{curv}}, \mathcal{O}(k+1)\right) \cong S^{k+1} H^{0}(S, E)^{*} .
$$

The construction of this isomorphism uses only Proposition 2 which remains true in our situation. Hence this step works as in [Voi02].

Fourth step. In [Voi02], we reduced easily the proof of the surjectivity of the map (7) in Lemma 1 to the proof of the following: let

$$
\widetilde{W}=W \times_{S^{[k+1]}} I_{k+1},
$$

and denote by $\gamma: \widetilde{W} \rightarrow W$ the natural map.

Proposition 3. The map

$$
\gamma^{*}: H^{0}\left(W, \psi^{*} L_{k+1}\right) \rightarrow H^{0}\left(\widetilde{W}, \gamma^{*} \psi^{*} L_{k+1}\right)
$$

is surjective.

Using the fact that

$$
\psi^{*} L_{k+1}=r^{*} \mathcal{O}_{\mathbb{P}\left(H^{0}(E)\right)_{\mathrm{curv}}}(k+1)
$$

where

$$
r=\beta \circ \gamma: \widetilde{W} \rightarrow \mathbb{P}\left(H^{0}(E)\right)_{\text {curv }},
$$

this proposition is a consequence of the following.

Proposition 4. The map

$$
r^{*}: H^{0}\left(\mathcal{O}_{\mathbb{P}\left(H^{0}(E)\right)_{\text {curv }}}(k+1)\right)=S^{k+1} H^{0}(S, E)^{*} \rightarrow H^{0}\left(\widetilde{W}, r^{*} \mathcal{O}_{\mathbb{P}\left(H^{0}(E)\right)_{\text {curv }}}(k+1)\right)
$$

is surjective.

It is in the proof of this proposition that we shall see a difference between the case considered in [Voi02] and the present case. Indeed, let us introduce as in [Voi02] the codimension 4 subscheme

$$
W^{\prime}=\left\{(z, \sigma) \in \widetilde{S \times S} \times \mathbb{P}\left(H^{0}(S, E)\right), \sigma_{\mid z}=0\right\},
$$

where $\widetilde{S \times S}$ is the blow-up of $S \times S$ along its diagonal, and hence parametrizes ordered length 2 subschemes of $S \times S$.

In [Voi02], we used the fact that $\widetilde{W}$ can be seen as a large (i.e. the complementary set has codimension $\geqslant 2$ ) Zariski open set in $W^{\prime}$, and the fact (which is inaccurately not mentioned explicitly) that $W^{\prime}$ is normal (in fact, it is smooth for $k+1>3$, see below) to conclude that

$$
H^{0}\left(\widetilde{W}, r^{*} \mathcal{O}_{\mathbb{P}\left(H^{0}(E)\right)_{\text {curv }}}(k+1)\right)=H^{0}\left(W^{\prime}, p r_{2}^{*} \mathcal{O}_{\mathbb{P}\left(H^{0}(S, E)\right)}(k+1)\right) .
$$

Here we cannot do that because it is no longer true that $W^{\prime}$ is normal, nor that $\widetilde{W}$ is large in $W^{\prime}$. In fact $W^{\prime}$ is not irreducible. Indeed, consider the rational curve $\Delta \subset S$. The exact sequence (4) together with the fact that $L_{\mid \Delta}^{\prime}$ is trivial shows that $E_{\mid \Delta}$ is trivial and that the restriction map

$$
H^{0}(S, E) \rightarrow H^{0}\left(\Delta, E_{\mid \Delta}\right)
$$

is surjective, the right-hand side being of rank 2. So $H^{0}(S, E(-\Delta))$ is of codimension 2 in $H^{0}(S, E)$, so that $W^{\prime}$ has one component isomorphic to $\Delta \times \Delta \times \mathbb{P}\left(H^{0}(S, E(-\Delta))\right)$.

However, what remains true in our situation is the following. 


\section{GREEN'S CANONICAL SYZYGY CONJECTURE FOR GENERIC CURVES OF ODD GENUS}

Lemma 2. Assume $k+1>3$ (Theorem 5 is already known for $k+1 \leqslant 3$ ). Let

$$
U:=\mathbb{P}\left(H^{0}(S, E)\right)-\mathbb{P}\left(H^{0}(S, E(-\Delta))\right) .
$$

Then $\left.W_{U}^{\prime}:=W^{\prime} \cap \widetilde{S \times S} \times U\right)$ is smooth and $\widetilde{W}$ is a large open set in it.

Proof. If a section of $E$ vanishes at a point of $\Delta$, then it vanishes along $\Delta$, hence its 0-locus is not zero-dimensional. So $\widetilde{W} \subset W^{\prime}$. The proof that it is a large open set is easy. To prove that $W_{U}^{\prime}$ is smooth, it suffices to show the following property.

Property 1. For any $z \in(S-\Delta)^{[2]}$, the restriction map

$$
H^{0}(S, E) \rightarrow H^{0}\left(E_{\mid z}\right)
$$

is surjective.

Choose a smooth curve $C^{\prime} \in\left|L^{\prime}\right|$ containing $z$. It exists because $z$ does not meet $\Delta$. There is an exact sequence

$$
0 \rightarrow D \rightarrow E_{\mid C^{\prime}} \rightarrow K_{C^{\prime}}-D \rightarrow 0,
$$

where $D$ is a divisor of degree $(k+2)$ on $C^{\prime}$, with $h^{0}(D)=2$. Furthermore, the map $H^{0}(S, E) \rightarrow$ $H^{0}\left(C^{\prime}, E_{\mid C^{\prime}}\right)$ is surjective and there is an exact sequence

$$
0 \rightarrow H^{0}\left(C^{\prime}, D\right) \rightarrow H^{0}\left(C^{\prime}, E_{\mid C^{\prime}}\right) \rightarrow H^{0}\left(C^{\prime}, K_{C^{\prime}}-D\right) \rightarrow 0 .
$$

Now, by Proposition 1, the curve $C^{\prime}$ is generic in the Brill-Noether sense. Hence, since $k+1>3$, it does not possess a $g_{k+4}^{2}$. Hence the map

$$
H^{0}\left(C^{\prime}, K_{C^{\prime}}-D\right) \rightarrow H^{0}\left(\left(K_{C^{\prime}}-D\right)_{\mid z}\right)
$$

is surjective. So the map (11) has at least rank 3, since $|D|$ has no base point so that the restriction map $H^{0}\left(C^{\prime}, D\right) \rightarrow H^{0}\left(D_{\mid z}\right)$ has at least rank 1 , and our statement will be proved if we can furthermore choose $C^{\prime}$ and $D$ so that the restriction map

$$
H^{0}\left(C^{\prime}, D\right) \rightarrow H^{0}\left(D_{\mid z}\right)
$$

is injective. Now take two sections $s, s^{\prime}$ of $E$ such that $d\left(s \wedge s^{\prime}\right)$ vanishes on $z$, but $s, s^{\prime}$ have independent restrictions in $H^{0}\left(E_{\mid z}\right)$. Such sections are easily shown to exist once we know that the restriction map (11) has rank at least 3. Let $C^{\prime}$ be defined by $d\left(s \wedge s^{\prime}\right)$. The sections $s, s^{\prime}$ generate a subline bundle $D$ of $E_{\mid C^{\prime}}$ as above and the two sections of $D$ restrict injectively to $z$.

Last step. Lemma 2 shows that we have an isomorphism

$$
H^{0}\left(\widetilde{W}, r^{*} \mathcal{O}_{\mathbb{P}\left(H^{0}(E)\right)_{\text {curv }}}(k+1)\right) \cong H^{0}\left(W_{U}^{\prime}, p r_{2}^{*} \mathcal{O}_{U}(k+1)\right),
$$

so that Proposition 4 reduces to the following.

Proposition 5. The map

$$
p r_{2}^{*}: H^{0}\left(U, \mathcal{O}_{U}(k+1)\right) \cong S^{k+1} H^{0}(S, E)^{*} \rightarrow H^{0}\left(W_{U}^{\prime}, p r_{2}^{*}\left(\mathcal{O}_{U}(k+1)\right)\right)
$$

is an isomorphism.

Proof. The proof works as in [Voi02]; we note that $W_{U}^{\prime}$ is the zero locus of a section $\tilde{\sigma}$ of a certain rank 4 vector bundle $p r_{1}^{*} \tilde{E}_{2} \otimes p r_{2}^{*}\left(\mathcal{O}_{U}(1)\right)$ on $\widetilde{S \times S} \times U$. We then use the corresponding Koszul resolution of $\mathcal{I}_{W_{U}^{\prime}}$ to conclude that

$$
H^{1}\left(\widetilde{S \times S} \times U, \mathcal{I}_{W_{U}^{\prime}} \otimes p_{2}^{*}\left(\mathcal{O}_{U}(k+1)\right)\right)=0 .
$$




\section{Voisin}

There is one difference with the case considered in [Voi02]: namely, the spectral sequence which converges to $H^{*}\left(\widehat{S \times S} \times U, \mathcal{I}_{W_{U}^{\prime}} \otimes p r_{2}^{*} \mathcal{O}_{U}(k+1)\right)$ has degree 1 terms,

$$
E_{1}^{i, 1-i}=H^{i}\left(\widetilde{S \times S} \times U, p r_{1}^{*} \bigwedge^{i} \tilde{E}_{2}^{*} \otimes p r_{2}^{*}\left(\mathcal{O}_{U}(k+1-i)\right)\right), \quad i \geqslant 1 .
$$

Of course we have

$$
H^{0}\left(U, \mathcal{O}_{U}(k+1)\right)=S^{k+1} H^{0}(S, E)^{*} .
$$

But, unlike the case considered in [Voi02], where we worked over the whole $\mathbb{P}\left(H^{0}(S, E)\right)$, there might be some terms

$$
H^{i-1}\left(\widetilde{S \times S}, \bigwedge^{i} \tilde{E}_{2}^{*}\right) \otimes H^{1}\left(U, \mathcal{O}_{U}(k+1)\right)
$$

contributing to the term $E_{1}^{i, 1-i}$ above. It turns out that this is not the case, due to the following lemma.

LEMMA 3. We have

$$
H^{i-1}\left(\widetilde{S \times S}, \bigwedge^{i} \tilde{E}_{2}^{*}\right)=0
$$

for any $i \geqslant 1$.

Proof. We refer to [Voi02, Proposition 6], for more details and similar computations.

First of all, the vanishing $h^{0}\left(\widetilde{S \times S}, \tilde{E}_{2}^{*}\right)=0$ follows from the fact that the dual vector bundle $\tilde{E}_{2}$ admits for the space of global sections the space $H^{0}(E)$, which generates it generically, and that all of these sections vanish somewhere.

Next, $\bigwedge^{4} \tilde{E}_{2}^{*} \cong \operatorname{det} \tilde{E}_{2}^{*}=(-L) \otimes(-L)(2 D)$, where $D$ is the exceptional divisor of $\widetilde{S \times S}$ and we denote abusively by $(-L) \otimes(-L)$ the pull-back of the line bundle $(-L) \otimes(-L)$ on $S \times S$ to $\widetilde{S \times S}$ via the blowing-down map. Since the canonical divisor of $\widetilde{S \times S}$ is equal to $D$, we get by Serre duality

$$
H^{3}\left(\widetilde{S \times S}, \bigwedge^{4} \tilde{E}_{2}^{*}\right)=H^{1}(\widetilde{S \times S}, L \otimes L(-D))^{*},
$$

and the space on the right is 0 because the multiplication map $S^{2} H^{0}(S, L) \rightarrow H^{0}(S, 2 L)$ is surjective.

To compute $H^{2}\left(\widetilde{S \times S}, \bigwedge^{3} \tilde{E}_{2}^{*}\right)$, we use the isomorphism

$$
\bigwedge^{3} \tilde{E}_{2}^{*} \cong \operatorname{det} \tilde{E}_{2}^{*} \otimes \tilde{E}_{2}
$$

and the exact sequence

$$
0 \rightarrow \tilde{E}_{2} \rightarrow p r_{1}^{*} E \oplus p r_{2}^{*} E \rightarrow \tau^{*} E \rightarrow 0
$$

where $\tau: D \rightarrow S$ is the restriction of the blowing-down map to $D$, and the $p r_{i}$ are the projections to $S$ composed with the blowing-down map. It then follows from the associated long exact sequence that we only have to prove the vanishings

$$
\begin{gathered}
H^{1}\left(D, \tau^{*} E(-2 L)\left(2 D_{\mid D}\right)\right)=0, \\
H^{2}\left(\widetilde{S \times S}, p_{i}^{*} E((-L) \otimes(-L))(2 D)\right)=0 .
\end{gathered}
$$

Equation (14) comes from the fact that $R^{1} \tau_{*}\left(2 D_{\mid D}\right) \cong \mathcal{O}_{S}$ and from $H^{0}(S, E(-2 L))=0$. Equation (15) is deduced from Serre's duality and from

$$
H^{2}\left(\widetilde{S \times S}, p r_{i}^{*} E^{*}(L \otimes L)(-D)\right)=0 .
$$




\section{GREEN'S CANONICAL SYZYGY CONJECTURE FOR GENERIC CURVES OF ODD GENUS}

This last property is itself deduced from $H^{2}\left(S \times S, p r_{i}^{*} E^{*}(L \otimes L)\right)=0$ and from

$$
H^{1}\left(D, p r_{i}^{*} E^{*}(L \otimes L)_{\mid D}\right)=H^{1}\left(D, \tau^{*}(E(2 L))\right)=0 .
$$

It only remains to prove the vanishing $H^{1}\left(\widetilde{S \times S}, \bigwedge^{2} \tilde{E}_{2}^{*}\right)=0$. We use for this the fact that $\bigwedge^{2} \tilde{E}_{2}^{*}$ has a filtration whose successive quotients are

$$
\bigwedge^{2}\left(p r_{1}^{*} E^{*} \oplus p r_{2}^{*} E^{*}\right),\left(p r_{1}^{*} E^{*} \oplus p r_{2}^{*} E^{*}\right) \otimes \tau^{*} E^{*} \otimes \mathcal{O}_{D}(D), \bigwedge^{2} \tau^{*} E^{*} \otimes \mathcal{O}_{D}(2 D),
$$

which follows from the dualization of the exact sequence (13) (cf. [Voi02]). It is immediate to prove that each term has $H^{1}=0$.

Once we have these vanishings, the spectral sequence converging to $H^{*}\left(\widetilde{S \times S} \times U, \mathcal{I}_{W_{U}^{\prime}} \otimes\right.$ $\left.p r_{2}^{*} \mathcal{O}_{U}(k+1)\right)$ has the same shape in degree 1 as in [Voi02], and then the proof of the vanishing (12) works as in [Voi02]. This concludes the proof of Proposition 5, and hence that of Theorem 5.

\section{Proof of Theorem 4}

We start recalling the duality theorem of [Gre84], which we state here only in the case of surfaces. Theorem 6 (Green). Let $X$ be a smooth projective surface, $M$ be a line bundle on $X$ which is generated by sections, and $\mathcal{F}$ be a coherent sheaf on $X$ satisfying the condition

$$
H^{1}(X, \mathcal{F}(s M))=0, \forall s \in \mathbb{Z} .
$$

Then there is, for all $p, q$, a duality isomorphism (which is canonical up to a multiplicative coefficient)

$$
K_{p, q}(X, M, \mathcal{F}) \cong K_{r-2-p, 3-q}\left(X, M, \mathcal{F}^{*} \otimes K_{X}\right)^{*},
$$

where $r+1=h^{0}(X, M)$.

We consider now the case where $X$ is the K3 surface $S$ of the previous section, $M$ is either $L$ or $L^{\prime}$, and $\mathcal{F}$ is trivial. Then, in the first case, $r+1=g+1=2 k+2$, and in the second case, $r^{\prime}+1=g^{\prime}+1=2 k+3$. So the duality theorem above gives, using the fact that $K_{S} \cong \mathcal{O}_{S}$,

$$
\begin{gathered}
K_{k, 1}(S, L)^{*} \cong K_{k-1,2}(S, L), \\
K_{k+1,1}\left(S, L^{\prime}\right)^{*} \cong K_{k-1,2}\left(S, L^{\prime}\right) .
\end{gathered}
$$

Theorem 5 now says that $K_{k+1,1}\left(S, L^{\prime}\right)=0$ or equivalently by (17)

$$
K_{k-1,2}\left(S, L^{\prime}\right)=0 \text {. }
$$

Next, recall that we want to prove that $K_{k, 1}(S, L)=0$, and by (16) this is equivalent to

$$
K_{k-1,2}(S, L)=0 \text {. }
$$

Recalling that $L^{\prime}=L+\Delta$, and choosing a $\sigma \in H^{0}\left(S, L^{\prime}\right)$ such that $\sigma$ generates $H^{0}\left(S, L^{\prime}\right) /$ $H^{0}(S, L)$, (equivalently, $\sigma$ nowhere vanishes along $\Delta$ ), we now have the following proposition.

Proposition 6. The space $K_{k-1,2}(S, L)$ is generated as follows: consider the Koszul differential

$$
\delta^{\prime}: \bigwedge^{k-1} H^{0}(S, L) \otimes H^{0}(S, L-\Delta) \rightarrow \bigwedge^{k-2} H^{0}(S, L) \otimes H^{0}(S, 2 L-\Delta) .
$$

For any $\alpha \in \operatorname{Ker} \delta^{\prime}$, multiplication on the right by $\sigma \in H^{0}(S, L+\Delta)$ provides an element $\alpha \cdot \sigma$ which is in

$$
\operatorname{Ker} \delta: \bigwedge^{k-1} H^{0}(S, L) \otimes H^{0}(S, 2 L) \rightarrow \bigwedge^{k-2} H^{0}(S, L) \otimes H^{0}(S, 3 L),
$$




\section{VoIsin}

where $\delta$ is also the Koszul differential but, however, acting on a different space. The classes of these elements $\alpha \cdot \sigma$ generate $K_{k-1,2}(S, L)$.

Proof. Let $\beta \in \operatorname{Ker} \delta \subset \bigwedge^{k-1} H^{0}(S, L) \otimes H^{0}(S, 2 L)$. Since we know that $K_{k-1,2}\left(S, L^{\prime}\right)=0$ by (18), we can write

$$
\beta=\delta \gamma
$$

for some $\gamma \in \bigwedge^{k} H^{0}\left(S, L^{\prime}\right) \otimes H^{0}\left(S, L^{\prime}\right)$. Since $H^{0}\left(S, L^{\prime}\right)=H^{0}(S, L) \oplus\langle\sigma\rangle$, we can now decompose $\gamma$ as

$$
\gamma=\gamma_{1}+\sigma \wedge \gamma_{2}+\gamma_{3} \otimes \sigma+\sigma \wedge \gamma_{4} \otimes \sigma
$$

where

$$
\begin{aligned}
\gamma_{1} \in \bigwedge^{k} H^{0}(S, L) \otimes H^{0}(S, L), & \gamma_{2} \in \bigwedge_{k-1}^{k-1} H^{0}(S, L) \otimes H^{0}(S, L), \\
\gamma_{3} \in \bigwedge^{k} H^{0}(S, L), & \gamma_{4} \in \bigwedge^{k-1} H^{0}(S, L) .
\end{aligned}
$$

The fact that $\delta \gamma=\beta$ belongs to $\bigwedge^{k-1} H^{0}(S, L) \otimes H^{0}(S, 2 L)$ implies that $\gamma_{4}=0$ since $\gamma_{4}$ identifies to the image of $\delta \gamma$ in $\bigwedge^{k-1} H^{0}\left(S, L^{\prime}\right) \otimes H^{0}\left(2 L_{\mid \Delta}^{\prime}\right)$. Next, since we consider $\beta$ only modulo

$$
\operatorname{Im} \delta: \bigwedge^{k} H^{0}(S, L) \otimes H^{0}(S, L) \rightarrow \bigwedge^{k-1} H^{0}(S, L) \otimes H^{0}(S, 2 L)
$$

we may assume, modifying $\beta$ by an exact element, that $\gamma_{1}=0$. Finally, we note that $\gamma$ is defined up to $\delta$-closed and in particular up to $\delta$-exact elements. Using the relation

$$
\gamma_{3} \otimes \sigma=-\delta\left(\sigma \wedge \gamma_{3}\right)-\sigma \wedge \delta \gamma_{3},
$$

we conclude that modifying $\gamma$ we may also assume that $\gamma_{3}=0$.

In conclusion, $K_{k-1,2}(S, L)$ is generated by classes of $\delta$-closed elements $\beta$ such that, in $\bigwedge^{k-1} H^{0}$ $\left(S, L^{\prime}\right) \otimes H^{0}\left(S, 2 L^{\prime}\right)$, we have

$$
\beta=\delta(\sigma \wedge \gamma)
$$

for some $\gamma \in \bigwedge^{k-1} H^{0}(S, L) \otimes H^{0}(S, L)$. Now we observe that the condition

$$
\delta(\sigma \wedge \gamma) \in \bigwedge^{k-1} H^{0}(S, L) \otimes H^{0}(S, 2 L)
$$

implies that $\delta \gamma=0$. Hence

$$
\delta(\sigma \wedge \gamma)=-\gamma \cdot \sigma
$$

The condition that

$$
\gamma \cdot \sigma \in \bigwedge^{k-1} H^{0}(S, L) \otimes H^{0}(S, 2 L)
$$

with $\sigma \in H^{0}(S, L+\Delta), \sigma_{\mid \Delta} \neq 0$, implies now that $\gamma \in \bigwedge^{k-1} H^{0}(S, L) \otimes H^{0}(S, L-\Delta)$. Hence $\gamma \in$ $\operatorname{Ker} \delta^{\prime}$ and the proposition is proved.

Our next task is to compute the dimension of the space

$$
K:=\operatorname{Ker}\left(\delta^{\prime}: \bigwedge^{k-1} H^{0}(S, L) \otimes H^{0}(S, L-\Delta) \rightarrow \bigwedge^{k-2} H^{0}(S, L) \otimes H^{0}(S, 2 L-\Delta)\right) .
$$

Note that the Koszul complex of $(S, L, L-\Delta)$ equipped with the Koszul differential $\delta^{\prime}$ has the 


\section{GREEN'S CANONICAL SYZYGY CONJECTURE FOR GENERIC CURVES OF ODD GENUS}

following shape:

$$
\begin{aligned}
0 \rightarrow \bigwedge^{k-1} H^{0}(S, L) \otimes H^{0}(S, L-\Delta) & \stackrel{\delta^{\prime}}{\rightarrow-2} \bigwedge^{k-i} H^{0}(S, L) \otimes H^{0}(S, 2 L-\Delta) \\
\cdots & \rightarrow \bigwedge^{0} H^{0}(S, L) \otimes H^{0}(S, i L-\Delta) \rightarrow \cdots
\end{aligned}
$$

So $K$ is the first cohomology group of this complex, while the next ones are the $K_{k-i, i-1}(S, L, L-\Delta)$ for $i \geqslant 2$. We now have the following lemma.

Lemma 4. The Koszul cohomology groups $K_{k-i, i-1}(S, L, L-\Delta)$ vanish for $i \geqslant 2$.

Proof. We observe that the triple $(S, L, L-\Delta)$ satisfies the assumptions of the duality Theorem 6 . Hence, using $K_{S} \cong \mathcal{O}_{S}$, and $h^{0}(L)=2 k+2$, we conclude that $K_{k-i, i-1}(S, L, L-\Delta)$ is dual to $K_{k-1+i, 3-i+1}(S, L,-L+\Delta)$.

If $i=2$, the last group is the cohomology at the middle of the sequence

$$
\bigwedge^{k+2} H^{0}(S, L) \otimes H^{0}(S, \Delta) \stackrel{\delta_{1}}{\longrightarrow} \bigwedge^{k+1} H^{0}(S, L) \otimes H^{0}(S, L+\Delta) \stackrel{\delta_{2}}{\longrightarrow} \bigwedge^{k} H^{0}(S, L) \otimes H^{0}(S, 2 L+\Delta) .
$$

Now we use the equality $L^{\prime}=L+\Delta$ and Theorem 5 to conclude that, if $\beta \in \operatorname{Ker} \delta_{2}$, then we have

$$
\beta=\delta \gamma,
$$

for some $\gamma \in \bigwedge^{k+2} H^{0}\left(S, L^{\prime}\right)$. As in the previous proof, we now write

$$
\gamma=\gamma_{1}+\sigma \wedge \gamma_{2}
$$

with

$$
\gamma_{1} \in \bigwedge^{k+2} H^{0}(S, L), \quad \gamma_{2} \in \bigwedge^{k+1} H^{0}(S, L)
$$

The fact that

$$
\delta \gamma=\beta \in \bigwedge^{k+1} H^{0}(S, L) \otimes H^{0}(S, L+\Delta)
$$

implies immediately that $\gamma_{2}=0$. Hence, in fact, we have in $\bigwedge^{k+1} H^{0}\left(S, L^{\prime}\right) \otimes H^{0}\left(S, L^{\prime}\right)$ the equality

$$
\beta=\delta \gamma, \quad \gamma \in \bigwedge^{k+2} H^{0}(S, L) .
$$

Using the fact that the inclusion $H^{0}(S, L) \subset H^{0}(S, L+\Delta)$ is the multiplication by the unique section of $H^{0}(S, \Delta)$, it is obvious that this is equivalent to $\beta \in \operatorname{Im} \delta_{1}$. So the claim is proved in this case.

Next assume that $i=3$. Then $K_{k-1+i, 3-i+1}(S, L,-L+\Delta)$ is the cohomology in the middle of the sequence

$$
\bigwedge^{k+3} H^{0}(S, L) \otimes H^{0}(S,-L+\Delta) \stackrel{\delta_{1}}{\longrightarrow} \bigwedge^{k+2} H^{0}(S, L) \otimes H^{0}(S, \Delta) \stackrel{\delta_{2}}{\longrightarrow} \bigwedge^{k+1} H^{0}(S, L) \otimes H^{0}(S, L+\Delta) .
$$

But since $H^{0}(S, \Delta)$ is of dimension 1 , it is easy to see that $\operatorname{Ker} \delta_{2}=0$. So this case is also proved.

Finally, if $i \geqslant 4, K_{k-1+i, 3-i+1}(S, L,-L+\Delta)$ is 0 because it is the cohomology at the middle of a complex with vanishing middle term, since $H^{0}(S, s L+\Delta)=\{0\}$ for $s<0$.

Corollary 1. The dimension of $K$ is equal to the binomial coefficient $\left(\begin{array}{c}2 k+1 \\ k-1\end{array}\right)$.

Proof. $K$ is the degree 0 cohomology group of the complex (21) whose higher-degree cohomology groups vanish. Hence the dimension of $K$ is equal to the Euler characteristic of this complex. Since the terms of the complex are $\bigwedge^{k-i} H^{0}(S, L) \otimes H^{0}(S, i L-\Delta)$ put in degree $i-1$, for $i \geqslant 1$, and since

$$
h^{0}(S, L)=2 k+2, h^{0}(S, i L-\Delta)=1+2 k i^{2}-2 i,
$$




\section{Voisin}

we are reduced to proving the following identity:

$$
\left(\begin{array}{c}
2 k+1 \\
k-1
\end{array}\right)=\sum_{i \geqslant 1}(-1)^{i-1}\left(\begin{array}{c}
2 k+2 \\
k-i
\end{array}\right)\left(1+2 k i^{2}-2 i\right) .
$$

The proof is left to the reader.

Now recall the vector bundle $E$ from (3). Our strategy to conclude the proof of Theorem 4, or equivalently the vanishing $K_{k-1,2}(S, L)=0$, will be to construct a map

$$
\phi: S^{k-1} H^{0}(S, E) \rightarrow K=\operatorname{Ker} \delta^{\prime}
$$

and to prove first of all that it is an isomorphism and secondly that all the elements in $\operatorname{Im} \phi$ are annihilated by the map $\cdot \sigma$ of Proposition 6 . The vanishing $K_{k-1,2}(S, L)=0$ will then be a consequence of Proposition 6.

Construction of $\phi$. Recall that $E_{\mid \Delta} \cong \mathcal{O}_{\Delta}^{2}$, and that the restriction map

$$
H^{0}(S, E) \rightarrow H^{0}\left(\Delta, E_{\mid \Delta}\right)
$$

is surjective. Since $H^{0}(S, E)$ is of dimension $k+3, H^{0}(S, E(-\Delta))$ is of dimension $k+1$. Consider the determinant map

$$
d: \bigwedge^{2} H^{0}(S, E) \rightarrow H^{0}\left(S, L^{\prime}\right)
$$

Note that for $v \in \bigwedge^{2} H^{0}(S, E(-\Delta))$, we have $d(v) \in H^{0}(S, L(-\Delta))$ and for $v \in H^{0}(S, E) \wedge$ $H^{0}(S, E(-\Delta))$, we have $d(v) \in H^{0}(S, L)$. Let

$$
w_{1}, \ldots, w_{k+1}
$$

be a basis of $H^{0}(S, E(-\Delta))$. The map $\phi$ is defined by the following formula:

$$
\phi\left(\tau^{k-1}\right)=\sum_{i<j}(-1)^{i+j} d\left(\tau \wedge w_{1}\right) \wedge \ldots \hat{i} \ldots \hat{j} \ldots \wedge d\left(\tau \wedge w_{k+1}\right) \otimes d\left(w_{i} \wedge w_{j}\right) .
$$

By the remarks above, we have

$$
\phi\left(\tau^{k-1}\right) \in \bigwedge^{k-1} H^{0}(S, L) \otimes H^{0}(S, L(-\Delta)) \subset \bigwedge^{k-1} H^{0}\left(S, L^{\prime}\right) \otimes H^{0}\left(S, L^{\prime}\right) .
$$

We now prove the following lemma.

Lemma 5. The image of $\phi$ is contained in $\operatorname{Ker} \delta^{\prime}$, where $\delta^{\prime}$ is the Koszul differential of (20).

Proof. Observe that we have the following quadratic equations for $S$, imbedded in the projective space via $\left|L^{\prime}\right|$ (these equations are in fact quadratic equations defining the Grassmannian of codimension 2 subspaces of $H^{0}(E)$, in which $S$ lies naturally): consider the natural map

$\psi: \bigwedge^{3} H^{0}(S, E) \otimes H^{0}(S, E) \rightarrow \bigwedge^{2} H^{0}(S, E) \otimes \bigwedge^{2} H^{0}(S, E) \stackrel{d \otimes d}{\longrightarrow} H^{0}\left(S, L^{\prime}\right) \otimes H^{0}\left(S, L^{\prime}\right) \rightarrow S^{2} H^{0}\left(S, L^{\prime}\right)$.

Here the first map sends $v_{1} \wedge v_{2} \wedge v_{3} \otimes \gamma$ to

$$
v_{2} \wedge v_{3} \otimes v_{1} \wedge \gamma-v_{1} \wedge v_{3} \otimes v_{2} \wedge \gamma+v_{1} \wedge v_{2} \otimes v_{3} \wedge \gamma
$$

We claim that the image of $\psi$ is contained in the ideal of $S$. The reason for this is simply that the map $\psi$ commutes with evaluation at $x \in S$ and that, since $\operatorname{rank} E=2$, we have $\bigwedge^{3} E_{x}=0$.

So we conclude that we have the following equalities:

$$
d\left(v_{2} \wedge v_{3}\right) \cdot d\left(v_{1} \wedge \gamma\right)-d\left(v_{1} \wedge v_{3}\right) \cdot d\left(v_{2} \wedge \gamma\right)+d\left(v_{1} \wedge v_{2}\right) \cdot d\left(v_{3} \wedge \gamma\right)=0 \quad \text { in } H^{0}\left(S, 2 L^{\prime}\right)
$$


We now compute

$$
\begin{aligned}
\delta^{\prime}\left(\phi\left(\tau^{k-1}\right)\right)= & \sum_{k<i<j}(-1)^{i+j+k} d\left(\tau \wedge w_{1}\right) \wedge \ldots \hat{k} \hat{i} \hat{j} \ldots \wedge d\left(\tau \wedge w_{k+1}\right) \otimes d\left(\tau \wedge w_{k}\right) \cdot d\left(w_{i} \wedge w_{j}\right) \\
& -\sum_{i<k<j}(-1)^{i+j+k} d\left(\tau \wedge w_{1}\right) \wedge \ldots \hat{i} \hat{k} \hat{j} \ldots \wedge d\left(\tau \wedge w_{k+1}\right) \otimes d\left(\tau \wedge w_{k}\right) \cdot d\left(w_{i} \wedge w_{j}\right) \\
& +\sum_{i<j<k}(-1)^{i+j+k} d\left(\tau \wedge w_{1}\right) \wedge \ldots \hat{i} \hat{j} \hat{k} \ldots \wedge d\left(\tau \wedge w_{k+1}\right) \otimes d\left(\tau \wedge w_{k}\right) \cdot d\left(w_{i} \wedge w_{j}\right) .
\end{aligned}
$$

This is also equal to

$$
\begin{aligned}
\sum_{i<j<k}(-1)^{i+j+k} d(\tau & \left.\wedge w_{1}\right) \wedge \ldots \hat{i} \ldots \hat{j} \ldots \hat{k} \ldots \wedge d\left(\tau \wedge w_{k+1}\right) \\
& \otimes\left(d\left(\tau \wedge w_{k}\right) \cdot d\left(w_{i} \wedge w_{j}\right)+d\left(\tau \wedge w_{i}\right) \cdot d\left(w_{j} \wedge w_{k}\right)-d\left(\tau \wedge w_{j}\right) \cdot d\left(w_{i} \wedge w_{k}\right)\right) .
\end{aligned}
$$

Hence, by (23), we find that

$$
\delta^{\prime}\left(\phi\left(\tau^{k-1}\right)\right)=0 \quad \text { in } \bigwedge^{k-2} H^{0}(S, L) \otimes H^{0}(S, 2 L-\Delta) .
$$

Remark 1. The map $\phi$ is strongly related to the construction due to Green and Lazarsfeld (see [Gre84, Appendix]) of nontrivial syzygies in $K_{r_{1}+r_{2}-1,1}\left(X, L_{1} \otimes L_{2}\right)$, where, for $i=1,2, L_{i}$ are line bundles on $X$ with $r_{i}+1=h^{0}\left(X, L_{i}\right)$. The precise relation is obtained by taking $X=C \in|L|$, $L_{1}$ a line bundle of degree $k+2$ on $C$ with $h^{0}\left(L_{1}\right)=2, h^{0}\left(L_{1}-\Delta_{\mid C}\right)=1$, and $L_{2}=K_{C}-L_{1}$. One has to use the relation (given by sequences like (4)) between the Lazarsfeld vector bundle $E$ and linear systems on the curve $C$, or more precisely $C \cup \Delta$.

We shall prove the following.

Proposition 7. The map

$$
\phi: S^{k-1} H^{0}(S, E) \rightarrow K
$$

is an isomorphism.

Proof. By Corollary 1, both spaces have the same dimension, since $\operatorname{dim} H^{0}(S, E)=k+3$. The fact that $\phi$ is an isomorphism reduces then to the following.

Proposition 8. The map $\phi$ is injective.

We postpone the proof of Proposition 8 to the next section.

Assuming Proposition 8, the proof of the vanishing

$$
K_{k-1,2}(S, L)=0
$$

is then a consequence of Proposition 7, Proposition 6 and of the following proposition.

Proposition 9. For $\beta \in \operatorname{Im} \phi \subset K$, we have

$$
\beta \cdot \sigma=0 \quad \text { in } K_{k-1,2}(S, L) .
$$

Proof. Let $\beta=\phi\left(\tau^{k-1}\right)$. We may assume first that $\tau \notin H^{0}(S, E(-\Delta))$, and then that $\sigma=d(\tau \wedge w)$, for some $w \in H^{0}(S, E)$, because the result depends only on the class of $\sigma$ modulo $H^{0}(S, L)$, and the map

$$
\begin{gathered}
H^{0}(S, E) \rightarrow H^{0}(S, L+\Delta) / H^{0}(S, L), \\
v \mapsto d(\tau \wedge v) \quad \bmod H^{0}(S, L)
\end{gathered}
$$




\section{VoIsin}

is surjective. Next recall the formula (22)

$$
\phi\left(\tau^{k-1}\right)=\sum_{i<j}(-1)^{i+j} d\left(\tau \wedge w_{1}\right) \wedge \ldots \hat{i} \ldots \hat{j} \ldots \wedge d\left(\tau \wedge w_{k+1}\right) \otimes d\left(w_{i} \wedge w_{j}\right) .
$$

Using (23) applied to $v_{1}=w_{i}, v_{2}=w_{j}, v_{3}=\tau, \gamma=w$, we now get

$$
d\left(w_{i} \wedge w_{j}\right) \cdot d(\tau \wedge w)=d\left(\tau \wedge w_{j}\right) \cdot d\left(w_{i} \wedge w\right)-d\left(\tau \wedge w_{i}\right) \cdot d\left(w_{j} \wedge w\right) .
$$

Hence

$$
\begin{aligned}
\phi\left(\tau^{k-1}\right) \cdot d(\tau \wedge w)= & \sum_{i<j}(-1)^{i+j} d\left(\tau \wedge w_{1}\right) \wedge \ldots \hat{i} \ldots \hat{j} \ldots \wedge d\left(\tau \wedge w_{k+1}\right) \\
& \otimes\left(-d\left(\tau \wedge w_{i}\right) \cdot d\left(w_{j} \wedge w\right)+d\left(\tau \wedge w_{j}\right) \cdot d\left(w_{i} \wedge w\right)\right) .
\end{aligned}
$$

Now the expression on the right is equal to $\delta \beta^{\prime}$, with

$$
\beta^{\prime}=\sum_{i}(-1)^{i} d\left(\tau \wedge w_{1}\right) \wedge \ldots \hat{i} \ldots \wedge d\left(\tau \wedge w_{k+1}\right) \otimes d\left(w_{i} \wedge w\right),
$$

and since $w_{i} \in H^{0}(S, E(-\Delta))$ we have

$$
d\left(\tau \wedge w_{i}\right) \in H^{0}(S, L), \quad d\left(w_{i} \wedge w\right) \in H^{0}(S, L)
$$

so that $\beta^{\prime} \in \bigwedge^{k} H^{0}(S, L) \otimes H^{0}(S, L)$. Hence $\beta \cdot \sigma=0$ in $K_{k-1,2}(S, L)$.

\section{Proof of Proposition 8}

Let us first recall the statement: we have the determinant map

$$
d: \bigwedge^{2} H^{0}(E) \rightarrow H^{0}\left(S, L^{\prime}\right)
$$

which has the property that it does not vanish on non-zero decomposable elements. Here the rank of $H^{0}(E)$ is $k+3$ and the rank of $H^{0}(E(-\Delta))$ is $k+1$. We defined the map

$$
\phi: S^{k-1} H^{0}(E) \rightarrow \bigwedge^{k-1} H^{0}(L) \otimes H^{0}(L(-\Delta))
$$

explicitly by the formula (cf. (22))

$$
\phi\left(\tau^{k-1}\right)=\sum_{i<j}(-1)^{i+j} d\left(\tau \wedge w_{1}\right) \wedge \ldots \hat{i} \ldots \hat{j} \ldots \wedge d\left(\tau \wedge w_{k+1}\right) \otimes d\left(w_{i} \wedge w_{j}\right),
$$

where the $w_{l}$ form a basis of $H^{0}(E(-\Delta))$. Proposition 8 states that this map is injective.

We give an ad hoc, presumably not optimal, proof of this, relying on the particular geometry of the determinant map $d$. We believe that it is in fact true for any $d$ satisfying the condition that $d$ does not vanish on decomposable elements.

We assume in the following that $k \geqslant 2$.

In our situation, let $x \in S$ be a generic point. Consider the composition $\phi_{x}$ of $\phi$ with the evaluation at $x$ :

$$
\phi_{x}: S^{k-1} H^{0}(E) \rightarrow \bigwedge^{k-1} H^{0}(S, L) \otimes H^{0}\left(S, L(-\Delta)_{\mid x}\right) .
$$

Choose the basis $w_{1}, \ldots, w_{k+1}$ in such a way that $w_{1}, \ldots, w_{k-1}$ form a basis of $H^{0}\left(S, E(-\Delta) \otimes \mathcal{I}_{x}\right)$. Then the $d\left(w_{i} \wedge w_{j}\right)$ vanish at $x$ if either $i$ or $j$ is not greater than $(k-1)$, while $d\left(w_{k} \wedge w_{k+1}\right)$ does not vanish in $H^{0}\left(S, L(-\Delta)_{\mid x}\right)$. Identifying this last space with $\mathbb{C}$, it follows that $\phi_{x}$ has the following form up to a coefficient:

$$
\phi_{x}\left(\tau^{k-1}\right)=d\left(\tau \wedge w_{1}\right) \wedge \cdots \wedge d\left(\tau \wedge w_{k-1}\right) .
$$




\section{GREEN'S CANONICAL SYZYGY CONJECTURE FOR GENERIC CURVES OF ODD GENUS}

First step. We first use formula (24) to express the map $\phi$, or rather its transpose, as the map induced in cohomology by the top exterior power of a vector bundle map over an adequate variety. This will allow us later on to use the Koszul resolution of such top exterior powers.

Denote by $\mathcal{V}$ the vector bundle on the $\mathrm{K} 3$ surface, which is defined by the exact sequence

$$
0 \rightarrow \mathcal{V} \rightarrow H^{0}(E(-\Delta)) \otimes \mathcal{O}_{S} \rightarrow E(-\Delta) \rightarrow 0 .
$$

So the fiber $\mathcal{V}_{x}$ at $x \in S$ is the space generated by the $w_{1}, \ldots, w_{k-1}$ introduced above.

On $Y:=\mathbb{P}\left(H^{0}(E)\right) \times S$, there is a natural map

$$
h: p r_{2}^{*} \mathcal{V} \otimes p r_{1}^{*} \mathcal{O}_{\mathbb{P}\left(H^{0}(E)\right)}(-1) \rightarrow H^{0}(S, L) \otimes \mathcal{O}_{Y},
$$

which at the point $(\tau, x)$ is the map

$$
d(\tau \wedge): H^{0}\left(E(-\Delta) \otimes \mathcal{I}_{x}\right) \rightarrow H^{0}(L) .
$$

This map is injective when $\tau \notin H^{0}\left(E(-\Delta) \otimes \mathcal{I}_{x}\right)$, and has for kernel $\langle\tau\rangle$ otherwise. Since we want to study the map induced in cohomology by the top exterior power of $h$, we first want to make $h$ into a morphism which is everywhere injective. This is done as follows (we refer to diagram (31) for the notation). Let

$$
\mathbb{P}(\mathcal{V})=: Z=\left\{(\tau, x) \in Y, \tau \in H^{0}\left(E(-\Delta) \otimes \mathcal{I}_{x}\right)\right\}
$$

So $Z$ is the locus of points $(\tau, x)$ where $h_{\tau, x}$ is not injective. Denote by $f: \tilde{Y} \rightarrow Y$ the blow-up of $Y$ along $Z$. For simplicity, denote by $\mathbb{P}$ the space $\mathbb{P}\left(H^{0}(E)\right)$ and by $p$ the map $p r_{1} \circ f: \tilde{Y} \rightarrow \mathbb{P}$. Also let $q:=p r_{2} \circ f: \widetilde{Y} \rightarrow S$. The map $h$ extends to a map

$$
\tilde{h}: \mathcal{G} \rightarrow H^{0}(S, L) \otimes \mathcal{O}_{\tilde{Y}},
$$

which is now injective everywhere, where $\mathcal{G}$ is obtained from $q^{*} \mathcal{V} \otimes p^{*} \mathcal{O}_{\mathbb{P}}(-1)$ by an elementary transform along the exceptional divisor $D$ of $f$. Namely, $\mathcal{G}$ fits in an exact sequence

$$
0 \rightarrow q^{*} \mathcal{V} \otimes p^{*} \mathcal{O}_{\mathbb{P}}(-1) \rightarrow \mathcal{G} \rightarrow \mathcal{H}_{D} \rightarrow 0,
$$

where $\mathcal{H}_{D}$ is a line bundle supported on $D$, and the restriction of the first map to $D$ has exactly for its kernel the kernel of the map $h_{\mid D}$, that is the sub-line bundle

$$
p^{*} \mathcal{O}_{\mathbb{P}}(-2)_{\mid D} \subset q^{*} \mathcal{V} \otimes p^{*} \mathcal{O}_{\mathbb{P}}(-1)_{\mid D} .
$$

Note that

$$
\bigwedge^{k-1} \mathcal{G}=\operatorname{det} \mathcal{G}=p^{*} \mathcal{O}_{\mathbb{P}}(-k+1) \otimes q^{*}\left(L^{-1}(\Delta)\right)(D)
$$

Let

$$
\tilde{h}_{k-1}: \operatorname{det} \mathcal{G} \rightarrow \bigwedge^{k-1} H^{0}(L) \otimes \mathcal{O}_{\widetilde{Y}}
$$

be the map which is the $(k-1)$ th exterior power of $\tilde{h}$, and let

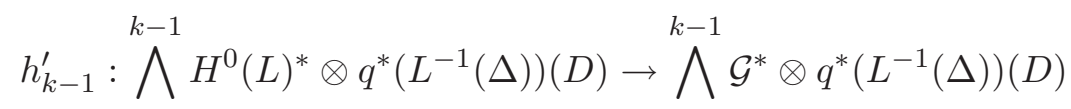

be the transpose of $\tilde{h}_{k-1}$ twisted by $q^{*}\left(L^{-1}(\Delta)\right)(D)$. We first claim that the transpose of the map $\phi$ identifies to the map $h^{2}\left(h_{k-1}^{\prime}\right)$ :

$$
\begin{array}{r}
H^{2}\left(\tilde{Y}, \bigwedge^{k-1} H^{0}(L)^{*} \otimes q^{*}\left(L^{-1}(\Delta)\right)(D)\right) \longrightarrow H^{2}\left(\tilde{Y}, \bigwedge^{k-1} \mathcal{G}^{*} \otimes q^{*}\left(L^{-1}(\Delta)\right)(D)\right) \\
\| \\
\bigwedge^{k-1} H^{0}(L)^{*} \otimes H^{0}(L(-\Delta))^{*} \stackrel{t_{\phi}}{\longrightarrow} H^{2}\left(\widetilde{Y}, p^{*} \mathcal{O}(k-1)\right)=S^{k-1} H^{0}(E)^{*} .
\end{array}
$$




\section{VoIsin}

Indeed, by (25), we have

$$
R^{k+2} q_{*}\left(K_{\widetilde{Y}} \otimes p^{*} \mathcal{O}_{\mathbb{P}}(-k+1)\right)=R^{k+2} q_{*}\left(K_{\widetilde{Y}} \otimes \operatorname{det} \mathcal{G} \otimes q^{*}(L(-\Delta))(-D)\right),
$$

and by Serre duality and $K_{S}=\mathcal{O}_{S}$, the left-hand side identifies to $S^{k-1} H^{0}(E) \otimes \mathcal{O}_{S}$. Now, formula (24) says that the map induced (up to a twist) by $\tilde{h}_{k-1}$,

$$
\begin{aligned}
S^{k-1} H^{0}(E) \otimes \mathcal{O}_{S} & \cong R^{k+2} q_{*}\left(K_{\widetilde{Y}} \otimes \operatorname{det} \mathcal{G} \otimes q^{*}(L(-\Delta))(-D)\right) \\
& \rightarrow R^{k+2} q_{*}\left(K_{\widetilde{Y}} \otimes \bigwedge^{k-1} H^{0}(L) \otimes q^{*}(L(-\Delta))(-D)\right)=\bigwedge H^{0}(L) \otimes L(-\Delta),
\end{aligned}
$$

is exactly the map $\phi$ followed with evaluation. Taking global sections on $S$, we conclude that $\phi$ is the map induced by $\tilde{h}_{k-1}$ (up to a twist),

$$
H^{k+2}\left(\widetilde{Y}, K_{\widetilde{Y}} \otimes \operatorname{det} \mathcal{G} \otimes q^{*}(L(-\Delta))(-D)\right) \rightarrow H^{k+2}\left(\widetilde{Y}, K_{\widetilde{Y}} \otimes \bigwedge^{k-1} H^{0}(L) \otimes q^{*}(L(-\Delta))(-D)\right),
$$

and applying Serre duality gives the result.

So the content of Proposition 8 is the surjectivity of the map $h^{2}\left(h_{k-1}^{\prime}\right)$.

Second step. We shall now analyse the spectral sequence associated to the Koszul resolution of Ker $h_{k-1}^{\prime}$ : associated to the surjective map ${ }^{t} \tilde{h}$, there is a resolution

$$
0 \rightarrow \bigwedge^{2 k+2} H^{0}(L)^{*} \otimes S^{k+3} \mathcal{G} \rightarrow \cdots \rightarrow \bigwedge^{k} H^{0}(L)^{*} \otimes \mathcal{G} \rightarrow \bigwedge_{k-1} H^{0}(L)^{*} \otimes \mathcal{O}_{\widetilde{Y}} \rightarrow \bigwedge^{k-1} \mathcal{G}^{*} \rightarrow 0
$$

We now claim that the surjectivity of the map (26) follows from the following lemmas.

Lemma 6 . For $1 \leqslant l \leqslant k$, or $l=k+2$, we have

$$
H^{l+2}\left(\widetilde{Y}, S^{l} \mathcal{G} \otimes q^{*}\left(L^{-1}(\Delta)\right)(D)\right)=0 .
$$

LEMMA 7. The sequence

$$
\begin{aligned}
\bigwedge^{2 k+1} H^{0}(L)^{*} \otimes H^{k+3}\left(\tilde{Y}, S^{k+2} \mathcal{G} \otimes q^{*}\left(L^{-1}(\Delta)\right)(D)\right) & \rightarrow \bigwedge^{2 k} H^{0}(L)^{*} \otimes H^{k+3}\left(\tilde{Y}, S^{k+1} \mathcal{G} \otimes q^{*}\left(L^{-1}(\Delta)\right)(D)\right) \\
& \rightarrow \bigwedge^{2 k-1} H^{0}(L)^{*} \otimes H^{k+3}\left(\tilde{Y}, S^{k} \mathcal{G} \otimes q^{*}\left(L^{-1}(\Delta)\right)(D)\right)
\end{aligned}
$$

induced by the complex (27) is exact at the middle.

Indeed, these two lemmas together imply that the vector bundle Ker $h_{k-1}^{\prime}$ satisfies the condition $H^{3}\left(\widetilde{Y}, \operatorname{Ker} h_{k-1}^{\prime}\right)=0$, which implies the surjectivity of $h^{2}\left(h_{k-1}^{\prime}\right)$. In fact, twisting the complex $(27)$ by $q^{*}\left(L^{-1}(\Delta)\right)(D)$, we get a resolution of Ker $h_{k-1}^{\prime}$ as follows:

$$
\begin{aligned}
0 \rightarrow \bigwedge^{2 k+2} H^{0}(L)^{*} \otimes S^{k+3} \mathcal{G} \otimes q^{*}\left(L^{-1}(\Delta)\right)(D) & \rightarrow \cdots \rightarrow \bigwedge^{k} H^{0}(L)^{*} \otimes \mathcal{G} \otimes q^{*}\left(L^{-1}(\Delta)\right)(D) \\
& \rightarrow \operatorname{Ker} h_{k-1}^{\prime} \rightarrow 0 .
\end{aligned}
$$

The associated spectral sequence abutting to the hypercohomology of the complex

$$
0 \rightarrow \bigwedge^{2 k+2} H^{0}(L)^{*} \otimes S^{k+3} \mathcal{G} \otimes q^{*}\left(L^{-1}(\Delta)\right)(D) \rightarrow \cdots \rightarrow \bigwedge^{k} H^{0}(L)^{*} \otimes \mathcal{G} \otimes q^{*}\left(L^{-1}(\Delta)\right)(D) \rightarrow 0,
$$

where we put the last term on the right-hand side in degree 0 , has

$$
E_{1}^{p, q} \cong \bigwedge^{k-p} H^{0}(L)^{*} \otimes H^{q}\left(\widetilde{Y}, S^{1-p} \mathcal{G} \otimes q^{*}\left(L^{-1}(\Delta)\right)(D)\right)
$$




\section{GREEN'S CANONICAL SYZYGY CONJECTURE FOR GENERIC CURVES OF ODD GENUS}

Now Lemma 6 says that the $E_{1}^{p, q}$ for $p+q=3, q \geqslant 3$ are 0 unless $q=k+3$. For $q=k+3$, we have $E_{1}^{-k, k+3} \neq 0$ but Lemma 7 says that $E_{2}^{-k, k+3}=0$. Hence this complex has $\mathbb{H}^{3}=0$ and thus $H^{3}\left(\widetilde{Y}, \operatorname{Ker} h_{k-1}^{\prime}\right)=0$. This proves the claim.

Third step. We now start proving Lemmas 6 and 7. We shall use for this another geometric definition of the vector bundle $\mathcal{G}$. We refer to diagram (31) for the notation.

Proof of Lemma 6. Let $\mathcal{F}$ be the quotient bundle $H^{0}(S, E) \otimes \mathcal{O}_{S} / \mathcal{V}$. There exists the relative projection

$$
\chi: \widetilde{Y} \rightarrow \mathbb{P}(\mathcal{F}),
$$

which makes $\tilde{Y}$ isomorphic to $\mathbb{P}(\mathcal{H})$, where $\mathcal{H}$ is a vector bundle on $\mathbb{P}(\mathcal{F})$ which fits in the exact sequence

$$
0 \rightarrow \pi^{*} \mathcal{V} \rightarrow \mathcal{H} \rightarrow \mathcal{O}_{\mathbb{P}(\mathcal{F})}(-1) \rightarrow 0
$$

where $\pi: \mathbb{P}(\mathcal{F}) \rightarrow S$ is the structural map. We now observe that $\mathcal{G}$ is naturally isomorphic to the twisted relative tangent bundle $T_{\chi} \otimes p^{*} \mathcal{O}_{\mathbb{P}}(-2)$. To see this, we consider the relative Euler sequence

$$
0 \rightarrow p^{*} \mathcal{O}_{\mathbb{P}}(-2) \rightarrow \chi^{*} \mathcal{H} \otimes p^{*} \mathcal{O}_{\mathbb{P}}(-1) \rightarrow T_{\chi}(-2) \rightarrow 0
$$

It induces a map

$$
q^{*} \mathcal{V} \otimes p^{*} \mathcal{O}_{\mathbb{P}}(-1) \rightarrow T_{\chi},
$$

and it follows from the Euler sequence that this map is injective away from $D$ and has $p^{*} \mathcal{O}_{\mathbb{P}}(-2)_{\mid D}$ as kernel along $D$. Hence $T_{\chi}$ is deduced from $q^{*} \mathcal{V} \otimes p^{*} \mathcal{O}_{\mathbb{P}}(-1)$ by the same elementary transform as $\mathcal{G}$.

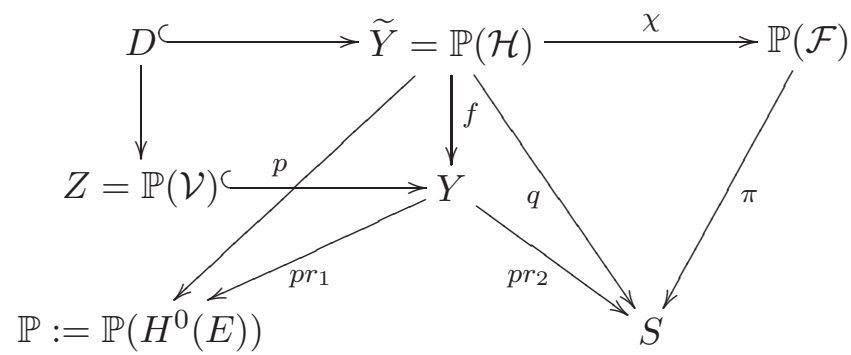

The relative Euler sequence (30) describes $\mathcal{G}$ by the exact sequence

$$
0 \rightarrow p^{*} \mathcal{O}_{\mathbb{P}}(-2) \rightarrow p^{*} \mathcal{O}_{\mathbb{P}}(-1) \otimes \chi^{*} \mathcal{H} \rightarrow \mathcal{G} \rightarrow 0 .
$$

Taking the $l$ th symmetric power, we get the exact sequence

$$
0 \rightarrow p^{*} \mathcal{O}_{\mathbb{P}}(-l-1) \otimes \chi^{*} S^{l-1} \mathcal{H} \rightarrow p^{*} \mathcal{O}_{\mathbb{P}}(-l) \otimes \chi^{*} S^{l} \mathcal{H} \rightarrow S^{l} \mathcal{G} \rightarrow 0 .
$$

Assume first that $1 \leqslant l \leqslant k-1$. By the exact sequence (33), the vanishing

$$
H^{l+2}\left(\tilde{Y}, S^{l} \mathcal{G} \otimes q^{*}\left(L^{-1}(\Delta)\right)(D)\right)=0
$$

is implied by the vanishings

$$
\begin{gathered}
H^{l+2}\left(\tilde{Y}, p^{*} \mathcal{O}_{\mathbb{P}}(-l) \otimes \chi^{*} S^{l} \mathcal{H} \otimes q^{*}\left(L^{-1}(\Delta)\right)(D)\right)=0, \\
H^{l+3}\left(\widetilde{Y}, p^{*} \mathcal{O}_{\mathbb{P}}(-l-1) \otimes \chi^{*} S^{l-1} \mathcal{H} \otimes q^{*}\left(L^{-1}(\Delta)\right)(D)\right)=0 .
\end{gathered}
$$

Now, if $k-1 \geqslant l \geqslant 2$, the line bundles $p^{*} \mathcal{O}_{\mathbb{P}}(-l)(D), p^{*} \mathcal{O}_{\mathbb{P}}(-l-1)(D)$ have trivial cohomology along the fibers of $\chi$, which are projective spaces $\mathbb{P}^{k-1}$, on which $D$ restricts to $\mathcal{O}(1)$. Hence the vanishings (34) are proved in this case. The case $l=1$ is also easy.

If $l=k$, the argument above gives an inclusion

$$
H^{k+2}\left(\tilde{Y}, S^{k} \mathcal{G} \otimes q^{*}\left(L^{-1}(\Delta)\right)(D)\right) \hookrightarrow H^{k+3}\left(\tilde{Y}, p^{*} \mathcal{O}_{\mathbb{P}}(-k-1) \otimes \chi^{*} S^{k-1} \mathcal{H} \otimes q^{*}\left(L^{-1}(\Delta)\right)(D)\right) .
$$




\section{VoIsin}

By Serre duality, this dualizes as

$$
H^{1}\left(\tilde{Y}, p^{*} \mathcal{O}_{\mathbb{P}}(-2) \otimes \chi^{*} S^{k-1} \mathcal{H}^{*} \otimes q^{*}(L(-\Delta))(2 D)\right) .
$$

Since $p^{*} \mathcal{O}_{\mathbb{P}}(-2)(2 D)=\chi^{*} \mathcal{O}_{\mathbb{P}(\mathcal{F})}(-2)$ and $S^{k-1} \mathcal{H}^{*}=R^{0} \chi_{*}\left(p^{*} \mathcal{O}_{\mathbb{P}}(k-1)\right)$, the last space is equal to

$$
H^{1}\left(\widetilde{Y}, p^{*} \mathcal{O}_{\mathbb{P}}(k-3)(2 D) \otimes q^{*}(L-(\Delta))\right),
$$

which is 0 because the map $f=(p, q)$ is the blow-down map, and $2 D$ has trivial cohomology along the fibers of $f$. It follows that

$$
H^{1}\left(\widetilde{Y}, p^{*} \mathcal{O}_{\mathbb{P}}(k-3)(2 D) \otimes q^{*}(L-(\Delta))\right)=H^{1}\left(Y, p r_{1}^{*} \mathcal{O}_{\mathbb{P}}(k-3) \otimes p r_{2}^{*}(L-(\Delta))\right)=0 .
$$

Next, if $l=k+2$, we use the inclusion $p^{*} \mathcal{O}_{\mathbb{P}}(-1) \otimes q^{*} \mathcal{V} \subset \mathcal{G}$, which is an isomorphism away from $D$, to get a surjective map

$$
H^{k+4}\left(\tilde{Y}, p^{*} \mathcal{O}_{\mathbb{P}}(-k-2) \otimes q^{*} S^{k+2} \mathcal{V} \otimes q^{*}\left(L^{-1}(\Delta)\right)(D)\right) \rightarrow H^{k+4}\left(\tilde{Y}, S^{k+2} \mathcal{G} \otimes q^{*}\left(L^{-1}(\Delta)\right)(D)\right) .
$$

The left-hand side is zero because $D$ has trivial cohomology along the fibers of $(p, q)$, so that

$$
\begin{aligned}
H^{k+4}\left(\tilde{Y}, p^{*} \mathcal{O}_{\mathbb{P}}(-k-2) \otimes q^{*} S^{k+2} \mathcal{V} \otimes q^{*}\right. & \left.\left(L^{-1}(\Delta)\right)(D)\right) \\
& =H^{k+4}\left(Y, p r_{1}^{*} \mathcal{O}_{\mathbb{P}}(-k-2) \otimes p r_{2}^{*}\left(S^{k+2} \mathcal{V} \otimes L^{-1}(\Delta)\right)=0\right.
\end{aligned}
$$

(recall that $Y=\mathbb{P}\left(H^{0}(E)\right) \times S$, with $\left.\operatorname{rank} H^{0}(E)=k+3\right)$. Hence we conclude that

$$
H^{k+4}\left(\tilde{Y}, S^{k+2} \mathcal{G} \otimes q^{*}\left(L^{-1}(\Delta)\right)(D)\right)=0 .
$$

In order to prove Lemma 7 , we will need the following lemma.

\section{LEMMA 8.}

(i) The space

$$
H^{k+3}\left(\tilde{Y}, S^{k+1} \mathcal{G} \otimes q^{*}\left(L^{-1}(\Delta)\right)(D)\right)
$$

is canonically isomorphic to

$$
S^{k-2} H^{0}(E) \otimes H^{2}\left(S, \mathcal{V} \otimes L^{-1}(\Delta)\right) .
$$

(ii) The space $H^{k+3}\left(\widetilde{Y}, S^{k+2} \mathcal{G} \otimes q^{*}\left(L^{-1}(\Delta)\right)(D)\right)$ is canonically isomorphic to

$$
S^{k-1} H^{0}(E) \otimes H^{2}\left(S, S^{2} \mathcal{V} \otimes L^{-1}(\Delta)\right) \text {. }
$$

(iii) The space $H^{k+3}\left(\tilde{Y}, S^{k} \mathcal{G} \otimes q^{*}\left(L^{-1}(\Delta)\right)(D)\right)$ is canonically isomorphic to

$$
S^{k-3} H^{0}(E) \otimes H^{2}\left(S, L^{-1}(\Delta)\right) .
$$

Proof. We use the exact sequence (33),

$$
0 \rightarrow \chi^{*} S^{l-1} \mathcal{H} \otimes p^{*} \mathcal{O}_{\mathbb{P}}(-l-1) \rightarrow \chi^{*} S^{l} \mathcal{H} \otimes p^{*} \mathcal{O}_{\mathbb{P}}(-l) \rightarrow S^{l} \mathcal{G} \rightarrow 0 .
$$

It implies by the associated long exact sequence that the space $H^{k+3}\left(\tilde{Y}, S^{l} \mathcal{G} \otimes q^{*}\left(L^{-1}(\Delta)\right)(D)\right)$ is isomorphic to

$$
\begin{aligned}
\operatorname{Ker}\left(H ^ { k + 4 } \left(\tilde{Y}, \chi^{*} S^{l-1} \mathcal{H} \otimes p^{*} \mathcal{O}_{\mathbb{P}}(-l-1) \otimes\right.\right. & \left.q^{*}\left(L^{-1}(\Delta)\right)(D)\right) \\
& \left.\rightarrow H^{k+4}\left(\widetilde{Y}, \chi^{*} S^{l} \mathcal{H} \otimes p^{*} \mathcal{O}_{\mathbb{P}}(-l) \otimes q^{*}\left(L^{-1}(\Delta)\right)(D)\right)\right) .
\end{aligned}
$$

Recalling that $p^{*} \mathcal{O}_{\mathbb{P}}(1)=\chi^{*} \mathcal{O}_{\mathbb{P}(\mathcal{F})}(1)(D)$, we can rewrite this as

$$
\begin{aligned}
\operatorname{Ker}\left(H ^ { k + 4 } \left(\tilde{Y}, \chi^{*} S^{l-1} \mathcal{H}\right.\right. & \left.\otimes \chi^{*} \mathcal{O}_{\mathbb{P}(\mathcal{F})}(-1) \otimes p^{*} \mathcal{O}_{\mathbb{P}}(-l) \otimes q^{*}\left(L^{-1}(\Delta)\right)\right) \\
& \left.\rightarrow H^{k+4}\left(\widetilde{Y}, \chi^{*} S^{l} \mathcal{H} \otimes \chi^{*} \mathcal{O}_{\mathbb{P}(\mathcal{F})}(-1) \otimes p^{*} \mathcal{O}_{\mathbb{P}}(-l+1) \otimes q^{*}\left(L^{-1}(\Delta)\right)\right)\right) .
\end{aligned}
$$




\section{GREEN'S CANONICAL SYZYGY CONJECTURE FOR GENERIC CURVES OF ODD GENUS}

Now, we use the formula

$$
K_{\tilde{Y} / \mathbb{P}(\mathcal{F})}=p^{*} \mathcal{O}_{\mathbb{P}}(-k) \otimes \chi^{*} \operatorname{det} \mathcal{H}^{*},
$$

that is, by the exact sequence $(29)$, which gives $\operatorname{det} \mathcal{H}^{*}=\pi^{*}(L(-\Delta)) \otimes \mathcal{O}_{\mathbb{P}(\mathcal{F})}(1)$,

$$
K_{\tilde{Y} / \mathbb{P}(\mathcal{F})}=p^{*} \mathcal{O}_{\mathbb{P}}(-l) \otimes \chi^{*} \mathcal{O}_{\mathbb{P}(\mathcal{F})(1)} \otimes q^{*}(L(-\Delta)) .
$$

It follows then by the Leray spectral sequence and relative Serre duality that (36) is also equal to

$$
\begin{aligned}
\operatorname{Ker}\left(H ^ { 5 } \left(\mathbb{P}(\mathcal{F}), S^{l-1} \mathcal{H} \otimes S^{l-k} \mathcal{H} \otimes\right.\right. & \left.\mathcal{O}_{\mathbb{P}(\mathcal{F})}(-2) \otimes \pi^{*}\left(L^{-2}(2 \Delta)\right)\right) \\
& \left.\rightarrow H^{5}\left(\mathbb{P}(\mathcal{F}), S^{l} \mathcal{H} \otimes S^{l-k-1} \mathcal{H} \otimes \mathcal{O}_{\mathbb{P}(\mathcal{F})}(-2) \otimes \pi^{*}\left(L^{-2}(2 \Delta)\right)\right)\right),
\end{aligned}
$$

where we make the convention that negative symmetric powers are zero, and where the map is induced by the natural map

$$
S^{l-1} \mathcal{H} \otimes S^{l-k} \mathcal{H} \rightarrow S^{l} \mathcal{H} \otimes S^{l-k-1} \mathcal{H}
$$

We now apply again relative Serre duality and the Leray spectral sequence to conclude that this is also equal to

$$
\begin{aligned}
\operatorname{Ker}\left(H ^ { k + 4 } \left(\tilde{Y}, p^{*} \mathcal{O}_{\mathbb{P}}(-k-l) \otimes\right.\right. & \left.\chi^{*} S^{l-k} \mathcal{H} \otimes q^{*}\left(L^{-1}(\Delta)\right)(D)\right) \\
& \left.\rightarrow H^{k+4}\left(\widetilde{Y}, p^{*} \mathcal{O}_{\mathbb{P}}(-k-l-1) \otimes \chi^{*} S^{l-k-1} \mathcal{H} \otimes q^{*}\left(L^{-1}(\Delta)\right)(D)\right)\right) .
\end{aligned}
$$

We now distinguish specific cases according to the value of $l$.

Case $l=k$. We have proved that in this case we have

$$
H^{k+3}\left(\widetilde{Y}, S^{k} \mathcal{G} \otimes q^{*}\left(L^{-1}(\Delta)\right)(D)\right) \cong H^{k+4}\left(\widetilde{Y}, p^{*} \mathcal{O}_{\mathbb{P}}(-2 k) \otimes q^{*}\left(L^{-1}(\Delta)\right)(D)\right) .
$$

Since $K_{\widetilde{Y}}=p^{*} \mathcal{O}_{\mathbb{P}}(-k-3)(3 D)$, this is Serre dual to

$$
H^{0}\left(\widetilde{Y}, p^{*} \mathcal{O}_{\mathbb{P}}(k-3) \otimes q^{*}(L(-\Delta))(2 D)\right)=S^{k-3} H^{0}(E)^{*} \otimes H^{0}(S, L(-\Delta)) .
$$

Applying Serre's duality on $S$ then gives Lemma 8(iii).

Case $l=k+1$. In this case, we have

$$
\begin{aligned}
H^{k+3}\left(\tilde{Y}, S^{k+1} \mathcal{G} \otimes q^{*}\left(L^{-1}(\Delta)\right)(D)\right) & \cong \operatorname{Ker}\left(H^{k+4}\left(\tilde{Y}, p^{*} \mathcal{O}_{\mathbb{P}}(-2 k-1) \otimes \chi^{*} \mathcal{H} \otimes q^{*}\left(L^{-1}(\Delta)\right)(D)\right)\right. \\
& \left.\rightarrow H^{k+4}\left(\tilde{Y}, p^{*} \mathcal{O}_{\mathbb{P}}(-2 k-2) \otimes q^{*}\left(L^{-1}(\Delta)\right)(D)\right)\right) .
\end{aligned}
$$

The right-hand side is computed as before: by Serre duality on $\widetilde{Y}$, we get

$$
H^{k+4}\left(\tilde{Y}, p^{*} \mathcal{O}_{\mathbb{P}}(-2 k-2) \otimes q^{*}\left(L^{-1}(\Delta)\right)(D)\right) \cong S^{k-1} H^{0}(E) \otimes H^{2}\left(S, L^{-1}(\Delta)\right) .
$$

To compute the left-hand side, we use the exact sequence (29):

$$
0 \rightarrow \pi^{*} \mathcal{V} \rightarrow \mathcal{H} \rightarrow \mathcal{O}_{\mathbb{P}(\mathcal{F})}(-1) \rightarrow 0 .
$$

Pulling back to $\tilde{Y}$ and tensoring with $p^{*} \mathcal{O}_{\mathbb{P}}(-2 k-1) \otimes q^{*}\left(L^{-1}(\Delta)\right)(D)$, it provides the exact sequence

$$
\begin{aligned}
0 & \rightarrow q^{*} \mathcal{V} \otimes p^{*} \mathcal{O}_{\mathbb{P}}(-2 k-1) \otimes q^{*}\left(L^{-1}(\Delta)\right)(D) \\
& \rightarrow \chi^{*} \mathcal{H} \otimes p^{*} \mathcal{O}_{\mathbb{P}}(-2 k-1) \otimes q^{*}\left(L^{-1}(\Delta)\right)(D) \\
& \rightarrow \chi^{*} \mathcal{O}_{\mathbb{P}(\mathcal{F})}(-1) \otimes p^{*} \mathcal{O}_{\mathbb{P}}(-2 k-1) \otimes q^{*}\left(L^{-1}(\Delta)\right)(D) \rightarrow 0 .
\end{aligned}
$$

Note that, as (29) is (non-canonically) split along the fibers of $\pi$, the sequence (39) is (noncanonically) split along the fibers of $q$. Hence there is an induced exact sequence on $S$ :

$$
\begin{aligned}
0 & \rightarrow \mathcal{V} \otimes L^{-1}(\Delta) \otimes R^{k+2} q_{*}\left(p^{*} \mathcal{O}_{\mathbb{P}}(-2 k-1)(D)\right) \\
& \rightarrow L^{-1}(\Delta) \otimes R^{k+2} q_{*}\left(\chi^{*} \mathcal{H} \otimes p^{*} \mathcal{O}_{\mathbb{P}}(-2 k-1)(D)\right) \\
& \rightarrow L^{-1}(\Delta) \otimes R^{k+2} q_{*}\left(\chi^{*} \mathcal{O}_{\mathbb{P}(\mathcal{F})}(-1) \otimes p^{*} \mathcal{O}_{\mathbb{P}}(-2 k-1)(D)\right) \rightarrow 0 .
\end{aligned}
$$




\section{VoIsin}

Using the equality

$$
K_{\widetilde{Y}}=K_{\widetilde{Y} / S}=p^{*} \mathcal{O}_{\mathbb{P}}(-k-3)(3 D)
$$

and relative Serre duality, we get

$$
\begin{aligned}
R^{k+2} q_{*}\left(p^{*} \mathcal{O}_{\mathbb{P}}(-2 k-1)(D)\right) & \cong R^{0} q_{*}\left(p^{*} \mathcal{O}_{\mathbb{P}}(k-2)(2 D)\right)^{*} \\
& =S^{k-2} H^{0}(E) \otimes \mathcal{O}_{S} .
\end{aligned}
$$

Similarly, we get

$$
\begin{aligned}
R^{k+2} q_{*}\left(p^{*} \mathcal{O}_{\mathbb{P}}(-2 k-1) \otimes \chi^{*} \mathcal{O}_{\mathbb{P}(\mathcal{F})}(-1)(D)\right) & =R^{k+2} q_{*}\left(p^{*} \mathcal{O}_{\mathbb{P}}(-2 k-2)(2 D)\right) \\
& \cong R^{0} q_{*}\left(p^{*} \mathcal{O}_{\mathbb{P}}(k-1)(D)\right)^{*} \\
& =S^{k-1} H^{0}(E) \otimes \mathcal{O}_{S} .
\end{aligned}
$$

The exact sequence (40) can thus be rewritten as

$$
\begin{aligned}
0 \rightarrow \mathcal{V} \otimes L^{-1}(\Delta) \otimes S^{k-2} H^{0}(E) & \rightarrow R^{k+2} q_{*}\left(\chi^{*} \mathcal{H} \otimes p^{*} \mathcal{O}_{\mathbb{P}}(-2 k-1)(D)\right) \\
& \rightarrow L^{-1}(\Delta) \otimes S^{k-1} H^{0}(E) \rightarrow 0 .
\end{aligned}
$$

This last exact sequence is now canonically split because

$$
H^{0}(S, \mathcal{V})=H^{1}(S, \mathcal{V})=0 .
$$

It follows that we have a canonical isomorphism

$$
\begin{aligned}
& H^{k+4}\left(\tilde{Y}, \chi^{*} \mathcal{H} \otimes p^{*} \mathcal{O}_{\mathbb{P}}(-2 k-1)(D)\right) \\
& \quad=H^{2}\left(S, R^{k+2} q_{*}\left(\chi^{*} \mathcal{H} \otimes p^{*} \mathcal{O}_{\mathbb{P}}(-2 k-1)(D)\right)\right) \\
& \quad=H^{2}\left(S, \mathcal{V} \otimes L^{-1}(\Delta)\right) \otimes S^{k-2} H^{0}(E) \oplus H^{2}\left(S, L^{-1}(\Delta)\right) \otimes S^{k-1} H^{0}(E) .
\end{aligned}
$$

In conclusion, using (37), (38) and (41), we have found a canonical identification

$$
\begin{aligned}
H^{k+3} & \left(\widetilde{Y}, S^{k+1} \mathcal{G} \otimes q^{*}\left(L^{-1}(\Delta)\right)(D)\right) \\
& \cong \operatorname{Ker}\left(S^{k-2} H^{0}(E) \otimes H^{2}\left(S, \mathcal{V} \otimes L^{-1}(\Delta)\right) \oplus S^{k-1} H^{0}(E) \otimes H^{2}\left(S, L^{-1}(\Delta)\right)\right. \\
& \left.\rightarrow S^{k-1} H^{0}(E) \otimes H^{2}\left(S, L^{-1}(\Delta)\right)\right) .
\end{aligned}
$$

One checks that the second component of the map is the identity, which gives a canonical identification

$$
H^{k+3}\left(\widetilde{Y}, S^{k+1} \mathcal{G} \otimes q^{*}\left(L^{-1}(\Delta)\right)(D)\right) \cong S^{k-2} H^{0}(E) \otimes H^{2}\left(S, \mathcal{V} \otimes L^{-1}(\Delta)\right),
$$

proving Lemma $8(\mathrm{i})$.

The isomorphism Lemma 8(ii) is proved in the same way.

Let us now compute the spaces $H^{2}\left(S, \mathcal{V} \otimes L^{-1}(\Delta)\right)$ and $H^{2}\left(S, S^{2} \mathcal{V} \otimes L^{-1}(\Delta)\right)$.

We first observe that the exact sequence

$$
0 \rightarrow \mathcal{V} \rightarrow H^{0}(E(-\Delta)) \otimes \mathcal{O}_{S} \rightarrow E(-\Delta) \rightarrow 0,
$$

and Serre duality give an isomorphism

$$
H^{2}\left(S, \mathcal{V} \otimes L^{-1}(\Delta)\right) \cong \operatorname{Ker} c
$$

where $c$ is the contraction map

$$
H^{0}(E(-\Delta)) \otimes H^{0}(S, L(-\Delta))^{*} \rightarrow H^{0}(E(-\Delta))^{*}
$$

induced by the determinant map

$$
d_{0}: \bigwedge^{2} H^{0}(E(-\Delta)) \rightarrow H^{0}(S, L(-\Delta))
$$




\section{GREEN'S CANONICAL SYZYGY CONJECTURE FOR GENERIC CURVES OF ODD GENUS}

Similarly the induced exact sequence

$$
0 \rightarrow S^{2} \mathcal{V} \rightarrow S^{2} H^{0}(E(-\Delta)) \otimes \mathcal{O}_{S} \rightarrow H^{0}(E(-\Delta)) \otimes E(-\Delta) \rightarrow \bigwedge^{2} E(-\Delta) \rightarrow 0
$$

gives a surjective map

$$
H^{2}\left(S, S^{2} \mathcal{V} \otimes L^{-1}(\Delta)\right) \rightarrow \operatorname{Ker} c^{\prime}
$$

where the contraction map

$$
c^{\prime}: S^{2} H^{0}(E(-\Delta)) \otimes H^{0}(S, L(-\Delta))^{*} \rightarrow H^{0}(E(-\Delta)) \otimes H^{0}(E(-\Delta))^{*}
$$

is also induced by $d_{0}$.

Using Lemma 8, we now rewrite the sequence (28) as follows. We first identify

$$
\bigwedge^{2 k+1} H^{0}(L)^{*}, \quad \bigwedge^{2 k} H^{0}(L)^{*}, \quad \bigwedge^{2 k-1} H^{0}(L)^{*}
$$

respectively to

$$
H^{0}(L), \quad \bigwedge^{2} H^{0}(L), \quad \bigwedge^{3} H^{0}(L) .
$$

Then, via the isomorphisms given in Lemma 8 and (42), (43) above, our sequence (28) becomes, after replacing the first term by its quotient given in (43) through which the first map factors,

$$
\begin{aligned}
H^{0}(L) \otimes S^{k-1} H^{0}(E) \otimes \operatorname{Ker} c^{\prime} & \rightarrow \bigwedge^{2} H^{0}(L) \otimes S^{k-2} H^{0}(E) \otimes \operatorname{Ker} c \\
& \rightarrow \bigwedge^{3} H^{0}(L) \otimes S^{k-3} H^{0}(E) \otimes H^{0}(S, L(-\Delta))^{*} .
\end{aligned}
$$

It is immediate to check that the maps of the complex are induced by the determinant map

$$
d: H^{0}(E) \otimes H^{0}(E(-\Delta)) \rightarrow H^{0}(L)
$$

and by the natural maps, for $(i, j)=(k-1,2),(k-2,1),(k-3,0)$,

$$
S^{i} H^{0}(E) \otimes S^{j} H^{0}(E(-\Delta)) \rightarrow S^{i-1} H^{0}(E) \otimes S^{j-1} H^{0}(E(-\Delta)) \otimes H^{0}(E) \otimes H^{0}(E(-\Delta)) .
$$

Fourth step. We now want to prove Lemma 7, which we have just proved to be equivalent to exactness at the middle of the sequence (44). At this point, we no longer need the K3 surface. We now use only the map $d$ and do geometry on the Grassmannian of subspaces of $H^{0}(E)$. We believe that this step is the only essential one in the proof of Proposition 8.

Denote by $G$ the Grassmannian of rank 2 subspaces of $H^{0}(E)$, and let $\mathcal{L}$ be the Plücker line bundle on $G, \mathcal{E}$ be the tautological rank 2 quotient bundle on $G$. Let $\widetilde{G}^{\prime}$ be the desingularization of the hypersurface $G^{\prime} \subset G$ parametrizing the $V \subset H^{0}(E)$ meeting $H^{0}(E(-\Delta)$ ), defined as

$$
\widetilde{G}^{\prime}=\left\{(v, V) \in \mathbb{P}\left(H^{0}(E(-\Delta))\right) \times G, v \in V\right\} .
$$

We shall also denote by $\mathcal{L}, \mathcal{E}$ the pull-backs of $\mathcal{L}, \mathcal{E}$ to $\widetilde{G}^{\prime}$ by the second projection. Let

$$
g: \widetilde{G}^{\prime} \rightarrow \mathbb{P} H^{0}(E(-\Delta))
$$

be the first projection, and denote by $H$ the line bundle $g^{*} \mathcal{O}_{\mathbb{P} H^{0}(E(-\Delta))}(1)$. Then, the map

$$
d_{0}: \bigwedge^{2} H^{0}(E(-\Delta)) \rightarrow H^{0}(L(-\Delta))
$$

allows one to define

$$
\begin{gathered}
I \subset \mathbb{P} H^{0}(L(-\Delta))^{*} \times \mathbb{P} H^{0}(E(-\Delta)), \\
I=\left\{(\sigma, v), \sigma\left(d_{0}(v \wedge \cdot)\right)=0 \text { in } H^{0}(E(-\Delta))^{*}\right\} .
\end{gathered}
$$




\section{VoIsin}

Let $\widetilde{I} \stackrel{\pi}{\rightarrow} \widetilde{G}^{\prime}$ be the fibered product $I \times_{\mathbb{P} H^{0}(E(-\Delta))} \widetilde{G}^{\prime}$. We shall denote by $\mathcal{K}$ the line bundle

$$
g^{\prime *}\left(p r_{1}^{*} \mathcal{O}_{\left.\mathbb{P} H^{0}(L(-\Delta))^{*}(1)\right)}\right.
$$

on $\widetilde{I}$, and by $\mathcal{L}^{\prime}, \mathcal{E}^{\prime}, H^{\prime}$ the pull-backs to $\widetilde{I}$ of the corresponding objects on $\widetilde{G}^{\prime}$, that is

$$
\mathcal{L}^{\prime}=\pi^{*} \mathcal{L}, \quad \mathcal{E}^{\prime}=\pi^{*} \mathcal{E}, \quad H^{\prime}=\pi^{*} H .
$$

The notation is summarized in the following diagram.

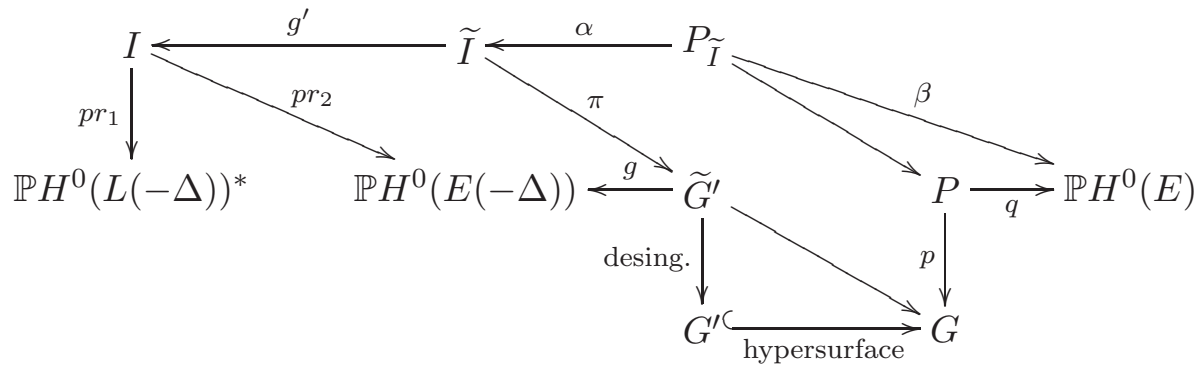

We shall use the following lemma.

Lemma 9. For any positive integers $p, s, t \geqslant s, p \geqslant s$, we have

$$
\begin{gathered}
H^{0}\left(\widetilde{I}, S^{p} \mathcal{E}^{\prime} \otimes \mathcal{L}^{\prime-s} \otimes H^{\prime t} \otimes \mathcal{K}\right) \cong H^{0}\left(\widetilde{I}, S^{p-s} \mathcal{E}^{\prime} \otimes H^{\prime t-s} \otimes \mathcal{K}\right), \\
H^{0}\left(\widetilde{I}, S^{p-s} \mathcal{E}^{\prime} \otimes H^{\prime t-s} \otimes \mathcal{K}\right) \cong S^{p-s} H^{0}(E)^{*} \otimes\left[S^{t-s} H^{0}(E(-\Delta)) \otimes H^{0}(L(-\Delta))^{*}\right]_{0}^{*} .
\end{gathered}
$$

Here, the term $\left[S^{t-s} H^{0}(E(-\Delta)) \otimes H^{0}(L(-\Delta))^{*}\right]_{0}$ has the following meaning: the map $d_{0}$ provides a contraction map

$$
H^{0}(E(-\Delta)) \otimes H^{0}(L(-\Delta))^{*} \rightarrow H^{0}(E(-\Delta))^{*}
$$

and more generally a contraction map

$$
c_{i}: S^{i} H^{0}(E(-\Delta)) \otimes H^{0}(L(-\Delta))^{*} \rightarrow S^{i-1} H^{0}(E(-\Delta)) \otimes H^{0}(E(-\Delta))^{*} .
$$

Then we denote

$$
\left[S^{i} H^{0}(E(-\Delta)) \otimes H^{0}(L(-\Delta))^{*}\right]_{0}:=\operatorname{Ker} c_{i} .
$$

Note that with the previous notation we have $c^{\prime}=c_{2}, c=c_{1}$.

Proof of Lemma 9. These facts are proved using the exact sequence on $\widetilde{G}^{\prime}$,

$$
0 \rightarrow \mathcal{L} \otimes H^{-1} \rightarrow \mathcal{E} \rightarrow H \rightarrow 0,
$$

or, pulling-back via $\pi$,

$$
0 \rightarrow \mathcal{L}^{\prime} \otimes H^{\prime-1} \rightarrow \mathcal{E}^{\prime} \rightarrow H^{\prime} \rightarrow 0
$$

on $\widetilde{I}$. Taking symmetric powers gives exact sequences

$$
0 \rightarrow S^{l-1} \mathcal{E}^{\prime} \otimes \mathcal{L}^{\prime} \otimes H^{\prime-1} \rightarrow S^{l} \mathcal{E}^{\prime} \rightarrow H^{\prime l} \rightarrow 0
$$

on $\widetilde{I}$. Taking $l=p$, tensoring (46) with $\mathcal{L}^{-s} \otimes H^{\prime t} \otimes \mathcal{K}$, and observing that if $s>0$,

$$
H^{0}\left(\widetilde{I}, \mathcal{L}^{\prime-s} \otimes H^{\prime p+t} \otimes \mathcal{K}\right)=0
$$

because the restriction of this line bundle to the fibers of $g^{\prime}$ is $\mathcal{O}(-s)$ on a projective space of dimension greater than 0 , we conclude that

$$
H^{0}\left(\widetilde{I}, S^{p} \mathcal{E}^{\prime} \otimes \mathcal{L}^{\prime-s} \otimes H^{\prime t} \otimes \mathcal{K}\right) \cong H^{0}\left(\widetilde{I}, S^{p-1} \mathcal{E}^{\prime} \otimes \mathcal{L}^{\prime-s+1} \otimes H^{\prime t-1} \otimes \mathcal{K}\right),
$$

which proves the first equality by iteration. 


\section{GREEN'S CANONICAL SYZYGY CONJECTURE FOR GENERIC CURVES OF ODD GENUS}

The second equality follows from the following observation: denoting by $P_{\widetilde{I}} \stackrel{(\alpha, \beta)}{\longrightarrow} \widetilde{I} \times \mathbb{P}\left(H^{0}(E)\right)$ the pull-back to $\widetilde{I}$ of the tautological $\mathbb{P}^{1}$-bundle $P$ on $G$ (see diagram (45)), there is a natural map

$$
P_{\widetilde{I}} \stackrel{\left(g^{\prime} \circ \alpha, \beta\right)}{\longrightarrow} I \times \mathbb{P}\left(H^{0}(E)\right)
$$

which is immediately seen to be birational. Furthermore, we have $S^{l} \mathcal{E}^{\prime} \cong R^{0} \alpha_{*}\left(\beta^{*} \mathcal{O}(l)\right)$ on $\widetilde{I}$, so that

$$
\begin{aligned}
H^{0}\left(\widetilde{I}, H^{\prime i} \otimes S^{j} \mathcal{E}^{\prime} \otimes \mathcal{K}\right) & =H^{0}\left(P_{\widetilde{I}}, \alpha^{*}\left(H^{\prime i} \otimes \mathcal{K}\right) \otimes \beta^{*} \mathcal{O}(j)\right) \\
& =H^{0}\left(P_{\widetilde{I}},\left(g^{\prime} \circ \alpha\right)^{*}\left(p r_{2}^{*} \mathcal{O}(i) \otimes \mathcal{K}\right) \otimes \beta^{*} \mathcal{O}(j)\right) .
\end{aligned}
$$

Because the map $\left(g^{\prime} \circ \alpha, \beta\right)$ is birational, this is also equal to

$$
H^{0}\left(I \times \mathbb{P}\left(H^{0}(E)\right), p r_{2}^{*} \mathcal{O}(i) \otimes \mathcal{K} \otimes \mathcal{O}(j)\right)=H^{0}\left(I, p r_{2}^{*} \mathcal{O}(i) \otimes \mathcal{K}\right) \otimes S^{j} H^{0}(E)^{*} .
$$

To conclude, it thus suffices to show the following equality:

$$
H^{0}\left(I, p r_{2}^{*} \mathcal{O}(i) \otimes \mathcal{K}\right)=\left[S^{i} H^{0}(E(-\Delta)) \otimes H^{0}(L(-\Delta))^{*}\right]_{0}^{*} .
$$

This last fact follows from the fact that, by definition of $I$ and $\mathcal{K}$, the vector bundle

$$
\mathcal{Q}:=R^{0} p r_{2 *} \mathcal{K}
$$

on $\mathbb{P} H^{0}(E(-\Delta))$ fits into the exact sequence

$$
0 \rightarrow \mathcal{O}(-2) \rightarrow \mathcal{O}(-1) \otimes H^{0}(E(-\Delta)) \stackrel{d_{0}}{\longrightarrow} H^{0}(L(-\Delta)) \otimes \mathcal{O} \rightarrow \mathcal{Q} \rightarrow 0 .
$$

It follows from this exact sequence, using the fact that $\operatorname{dim} H^{0}(E(-\Delta))=k+1 \geqslant 3$ and vanishing on $\mathbb{P} H^{0}(E(-\Delta))$, that

$$
\begin{aligned}
H^{0}\left(I, p r_{2}^{*} \mathcal{O}(i) \otimes \mathcal{K}\right) & =H^{0}\left(\mathbb{P} H^{0}(E(-\Delta)), \mathcal{O}(i) \otimes R^{0} p r_{2 *} \mathcal{K}\right) \\
& =H^{0}\left(\mathbb{P} H^{0}(E(-\Delta)), \mathcal{O}(i) \otimes \mathcal{Q}\right)
\end{aligned}
$$

is equal to the cokernel of the map induced by $d_{0}$,

$$
S^{i-1} H^{0}(E(-\Delta))^{*} \otimes H^{0}(E(-\Delta)) \rightarrow S^{i} H^{0}(E(-\Delta))^{*} \otimes H^{0}(L(-\Delta)),
$$

that is to $\left[S^{i} H^{0}(E(-\Delta)) \otimes H^{0}(L(-\Delta))^{*}\right]_{0}^{*}$.

This lemma will provide, in particular, canonical identifications

$$
\begin{aligned}
H^{0}\left(\widetilde{I}, S^{k-2} \mathcal{E}^{\prime} \otimes \mathcal{K} \otimes\right. & \left.H^{\prime} \otimes \mathcal{L}^{\prime-1}\right)=S^{k-3} H^{0}(E)^{*} \otimes H^{0}(L(-\Delta)), \\
H^{0}\left(\widetilde{I}, S^{k-2} \mathcal{E}^{\prime} \otimes \mathcal{K} \otimes H^{\prime}\right) * & =S^{k-2} H^{0}(E)^{*} \otimes\left[H^{0}(E(-\Delta)) \otimes H^{0}(L(-\Delta))^{*}\right]_{0}^{*} \\
& =S^{k-2} H^{0}(E)^{*} \otimes(\operatorname{Ker} c)^{*},
\end{aligned}
$$

an inclusion

$$
H^{0}\left(\widetilde{I}, S^{k-2} \mathcal{E}^{\prime} \otimes \mathcal{K} \otimes H^{\prime} \otimes \mathcal{L}^{\prime}\right) \subset H^{0}\left(\widetilde{I}, S^{k-1} \mathcal{E}^{\prime} \otimes \mathcal{K} \otimes H^{\prime 2}\right)
$$

and an identification

$$
\begin{aligned}
H^{0}\left(\widetilde{I}, S^{k-1} \mathcal{E}^{\prime} \otimes \mathcal{K} \otimes H^{\prime 2}\right) & =S^{k-1} H^{0}(E)^{*} \otimes\left[S^{2} H^{0}(E(-\Delta)) \otimes H^{0}(L(-\Delta))^{*}\right]_{0}^{*} \\
& =S^{k-1} H^{0}(E)^{*} \otimes\left(\operatorname{Ker} c^{\prime}\right)^{*} .
\end{aligned}
$$

They are used as follows. The determinant map $d: H^{0}(E) \otimes H^{0}(E(-\Delta)) \rightarrow H^{0}(L)$ provides dually a linear system

$$
H^{0}(L)^{*}=: W \subset H^{0}\left(G^{\prime}, \mathcal{L}\right) \subset H^{0}\left(\widetilde{I}, \mathcal{L}^{\prime}\right)
$$

which has no base point by Proposition 2. This provides an exact Koszul complex on $\widetilde{I}$ :

$$
0 \rightarrow \bigwedge^{2 k+2} W \otimes \mathcal{L}^{\prime-2 k-2} \rightarrow \cdots \rightarrow W \otimes \mathcal{L}^{\prime-1} \rightarrow \mathcal{O}_{\widetilde{I}} \rightarrow 0
$$




\section{VoIsin}

We twist by $S^{k-2} \mathcal{E}^{\prime} \otimes \mathcal{L}^{\prime \otimes 2} \otimes H^{\prime} \otimes \mathcal{K}$ and take global sections. The relevant piece of this complex of global sections is

$$
\begin{aligned}
\cdots & \rightarrow \bigwedge^{3} W \otimes H^{0}\left(\widetilde{I}, S^{k-2} \mathcal{E}^{\prime} \otimes \mathcal{K} \otimes H^{\prime} \otimes \mathcal{L}^{\prime-1}\right) \rightarrow \\
\bigwedge^{2} W \otimes H^{0}\left(\widetilde{I}, S^{k-2} \mathcal{E}^{\prime} \otimes \mathcal{K} \otimes H^{\prime}\right) & \rightarrow W \otimes H^{0}\left(\widetilde{I}, S^{k-2} \mathcal{E}^{\prime} \otimes \mathcal{K} \otimes H^{\prime} \otimes \mathcal{L}^{\prime}\right) \rightarrow \cdots .
\end{aligned}
$$

Using the inclusion (50), this sequence has the same cohomology at the middle as the sequence:

$$
\begin{aligned}
\cdots & \rightarrow \bigwedge^{3} W \otimes H^{0}\left(\widetilde{I}, S^{k-2} \mathcal{E}^{\prime} \otimes \mathcal{K} \otimes H^{\prime} \otimes \mathcal{L}^{\prime-1}\right) \rightarrow \\
\bigwedge^{2} W \otimes H^{0}\left(\widetilde{I}, S^{k-2} \mathcal{E}^{\prime} \otimes \mathcal{K} \otimes H^{\prime}\right) & \rightarrow W \otimes H^{0}\left(\widetilde{I}, S^{k-1} \mathcal{E}^{\prime} \otimes \mathcal{K} \otimes H^{\prime 2}\right) \rightarrow \cdots .
\end{aligned}
$$

Finally, using the identifications (48), (49) and (51) above, we see that the three terms of this last sequence are canonically dual to the three terms of the sequence (44). We leave it to the reader to verify that this last sequence is indeed dual to (44). Hence, the exactness at the middle of (44) is equivalent to the exactness at the middle of the Koszul sequence (53), and we claim that this is implied by the following statement.

Lemma 10. For $1 \leqslant i \leqslant 2 k-1$ we have the vanishing

$$
H^{i}\left(\widetilde{I}, S^{k-2} \mathcal{E}^{\prime} \otimes \mathcal{K} \otimes H^{\prime} \otimes \mathcal{L}^{\prime-i-1}\right)=0
$$

Indeed, the Koszul complex (52) twisted by $S^{k-2} \mathcal{E}^{\prime} \otimes \mathcal{K} \otimes H^{\prime} \otimes \mathcal{L}^{\prime 2}$ reads

$$
\begin{aligned}
0 & \rightarrow \bigwedge^{2 k+2} W \otimes S^{k-2} \mathcal{E}^{\prime} \otimes \mathcal{K} \otimes H^{\prime} \otimes \mathcal{L}^{\prime-2 k} \rightarrow \cdots \\
& \rightarrow \bigwedge^{2} W \otimes S^{k-2} \mathcal{E}^{\prime} \otimes \mathcal{K} \otimes H^{\prime} \rightarrow W \otimes S^{k-2} \mathcal{E}^{\prime} \otimes \mathcal{K} \otimes H^{\prime} \otimes \mathcal{L}^{\prime} \rightarrow \cdots
\end{aligned}
$$

In particular, this provides by truncation a resolution

$$
0 \rightarrow \bigwedge^{2 k+2} W \otimes S^{k-2} \mathcal{E}^{\prime} \otimes \mathcal{K} \otimes H^{\prime} \otimes \mathcal{L}^{\prime-2 k} \rightarrow \cdots \rightarrow \bigwedge^{4} W \otimes S^{k-2} \mathcal{E}^{\prime} \otimes \mathcal{K} \otimes H^{\prime} \otimes \mathcal{L}^{\prime-2} \rightarrow \mathcal{M} \rightarrow 0
$$

of the sheaf $\mathcal{M}$ on $\widetilde{I}$ which fits in the exact sequence

$$
\begin{aligned}
0 \rightarrow \mathcal{M} & \rightarrow \bigwedge^{3} W \otimes S^{k-2} \mathcal{E}^{\prime} \otimes \mathcal{K} \otimes H^{\prime} \otimes \mathcal{L}^{\prime-1} \\
& \rightarrow \bigwedge^{2} W \otimes S^{k-2} \mathcal{E}^{\prime} \otimes \mathcal{K} \otimes H^{\prime} \rightarrow W \otimes S^{k-2} \mathcal{E}^{\prime} \otimes \mathcal{K} \otimes H^{\prime} \otimes \mathcal{L}^{\prime} \cdots
\end{aligned}
$$

Now the exactness at the middle of the sequence $(53)$ is implied by the vanishing $H^{1}(\widetilde{I}, \mathcal{M})=0$. By the above resolution, this space is isomorphic to the hypercohomology group

$$
\mathbb{H}^{1}\left(\tilde{I}, 0 \rightarrow \bigwedge^{2 k+2} W \otimes S^{k-2} \mathcal{E}^{\prime} \otimes \mathcal{K} \otimes H^{\prime} \otimes \mathcal{L}^{\prime-2 k} \rightarrow \cdots \rightarrow \bigwedge^{4} W \otimes S^{k-2} \mathcal{E}^{\prime} \otimes \mathcal{K} \otimes H^{\prime} \otimes \mathcal{L}^{\prime-2} \rightarrow 0\right),
$$

where the last term on the right-hand side is put in degree 0 . The terms $E_{1}^{p, q}$ of the spectral sequence associated to the naive filtration of this complex are equal to

$$
\bigwedge^{4-p} W \otimes H^{q}\left(\widetilde{I}, S^{k-2} \mathcal{E}^{\prime} \otimes \mathcal{K} \otimes H^{\prime} \otimes \mathcal{L}^{\prime p-2}\right)
$$




\section{GREEN'S CANONICAL SYZYGY CONJECTURE FOR GENERIC CURVES OF ODD GENUS}

in degree $(p+q)$, that is, for $p+q=1$, to

$$
\bigwedge^{q+3} W \otimes H^{q}\left(\widetilde{I}, S^{k-2} \mathcal{E}^{\prime} \otimes \mathcal{K} \otimes H^{\prime} \otimes \mathcal{L}^{\prime-q-1}\right), \quad q \geqslant 1 .
$$

Hence the vanishings (55) say that the terms $E_{1}^{p, q}, p+q=1$, of this spectral sequence are 0 for $1 \leqslant q \leqslant 2 k-1$ and they are also obviously 0 for $q>2 k-1$, since then $\wedge^{q+3} W=0$. Thus $(57)$ vanishes and so does $H^{1}(\widetilde{I}, \mathcal{M})$. This proves the claim.

Proof of Lemma 10. Consider the following Cartesian diagram (see diagram (45)).

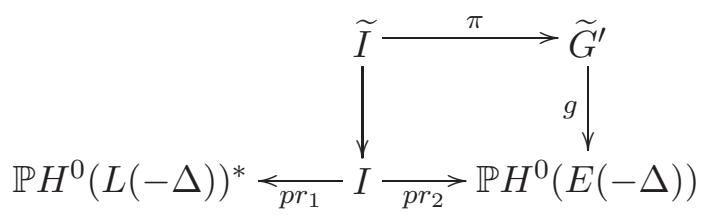

We have

$$
R^{0} \pi_{*} \mathcal{K}=g^{*}\left(R^{0} p r_{2 *}\left(p r_{1}^{*} \mathcal{O}(1)\right)\right)=g^{*} \mathcal{Q},
$$

where the bundle $\mathcal{Q}$ on $\mathbb{P} H^{0}(E(-\Delta))$ admits the resolution (see (47))

$$
0 \rightarrow \mathcal{O}(-2) \rightarrow \mathcal{O}(-1) \otimes H^{0}(E(-\Delta)) \stackrel{d_{0}}{\longrightarrow} H^{0}(L(-\Delta)) \otimes \mathcal{O} \rightarrow \mathcal{Q} \rightarrow 0 .
$$

Since $\mathcal{E}^{\prime}, \mathcal{L}^{\prime}, H^{\prime}$ are pull-backs via $\pi$ of the corresponding objects on $\widetilde{G}^{\prime}$, we have

$$
H^{i}\left(\widetilde{I}, S^{k-2} \mathcal{E}^{\prime} \otimes \mathcal{K} \otimes H^{\prime} \otimes \mathcal{L}^{\prime-i-1}\right)=H^{i}\left(\widetilde{G}^{\prime}, S^{k-2} \mathcal{E} \otimes H \otimes \mathcal{L}^{-i-1} \otimes g^{*} \mathcal{Q}\right) .
$$

The bundle $g^{*} \mathcal{Q}$ admits the resolution

$$
0 \rightarrow H^{-2} \rightarrow H^{-1} \otimes H^{0}(E(-\Delta)) \stackrel{d_{0}}{\longrightarrow} H^{0}(L(-\Delta)) \otimes \mathcal{O}_{\widetilde{G}^{\prime}} \rightarrow g^{*} \mathcal{Q} \rightarrow 0,
$$

and it follows that the desired vanishing

$$
H^{i}\left(\widetilde{G}^{\prime}, S^{k-2} \mathcal{E} \otimes H \otimes \mathcal{L}^{-i-1} \otimes g^{*} \mathcal{Q}\right)=0, \quad 1 \leqslant i \leqslant 2 k-1
$$

is a consequence of

$$
\begin{aligned}
H^{i}\left(\widetilde{G}^{\prime}, S^{k-2} \mathcal{E} \otimes H \otimes \mathcal{L}^{-i-1}\right)=0, & 1 \leqslant i \leqslant 2 k-1, \\
H^{i+1}\left(\widetilde{G}^{\prime}, S^{k-2} \mathcal{E} \otimes \mathcal{L}^{-i-1}\right)=0, & 1 \leqslant i \leqslant 2 k-1, \\
H^{i+2}\left(\widetilde{G}^{\prime}, S^{k-2} \mathcal{E} \otimes H^{-1} \otimes \mathcal{L}^{-i-1}\right)=0, & 1 \leqslant i \leqslant 2 k-2,
\end{aligned}
$$

and the following fact.

Lemma 11. The map

$$
H^{i+2}\left(\widetilde{G}^{\prime}, S^{k-2} \mathcal{E} \otimes H^{-1} \otimes \mathcal{L}^{-i-1}\right) \rightarrow H^{0}(E(-\Delta)) \otimes H^{i+2}\left(\widetilde{G}^{\prime}, S^{k-2} \mathcal{E} \otimes \mathcal{L}^{-i-1}\right)
$$

induced by the natural inclusion

$$
H^{-1} \subset H^{0}(E(-\Delta)) \otimes \mathcal{O}_{\widetilde{G}^{\prime}}
$$

is injective for $i=2 k-1$.

Let us first prove (59). We use the fact that we can see $\widetilde{G}^{\prime}$ as the complete intersection of two members of $|H|$ on the tautological $\mathbb{P}^{1}$-bundle $P$ on $G$. Indeed, let $P \subset G \times \mathbb{P} H^{0}(E)$ be the tautological subbundle, and denote by $p: P \rightarrow G$ the first projection and by $q: P \rightarrow \mathbb{P} H^{0}(E)$ the second projection (see diagram (45)). Denote by $H$ the line bundle $q^{*} \mathcal{O}(1)$ on $P$, and by $\mathcal{E}, \mathcal{L}$ the pull-backs via $p$ of the corresponding bundles on $G$. Then, by definition, $\widetilde{G}^{\prime}$ identifies to $q^{-1}\left(\mathbb{P} H^{0}(E(-\Delta))\right)$, and the bundles $H, \mathcal{E}, \mathcal{L}$ are the restrictions to $\widetilde{G}^{\prime}$ of the corresponding objects on $P$. 


\section{VoIsin}

Hence there is a Koszul resolution of $\mathcal{O}_{\widetilde{G}^{\prime}}$ which has the form

$$
0 \rightarrow \bigwedge^{2} R \otimes H^{-2} \rightarrow R \otimes H^{-1} \rightarrow \mathcal{O}_{P} \rightarrow \mathcal{O}_{\widetilde{G}^{\prime}} \rightarrow 0
$$

where $R$ is a rank 2 vector space.

Using this resolution, we see that the vanishing statements (59) are a consequence of the following:

(i) $H^{i}\left(P, S^{k-2} \mathcal{E} \otimes H \otimes \mathcal{L}^{-i-1}\right)=0, \quad 1 \leqslant i \leqslant 2 k-1$;

(ii) $H^{i+1}\left(P, S^{k-2} \mathcal{E} \otimes \mathcal{L}^{-i-1}\right)=0, \quad 1 \leqslant i \leqslant 2 k-1$;

(iii) $H^{i+2}\left(P, S^{k-2} \mathcal{E} \otimes H^{-1} \otimes \mathcal{L}^{-i-1}\right)=0, \quad 1 \leqslant i \leqslant 2 k-1$;

(iv) $H^{i+3}\left(P, S^{k-2} \mathcal{E} \otimes H^{-2} \otimes \mathcal{L}^{-i-1}\right)=0, \quad 1 \leqslant i \leqslant 2 k-1$;

(v) $H^{i+4}\left(P, S^{k-2} \mathcal{E} \otimes H^{-3} \otimes \mathcal{L}^{-i-1}\right)=0, \quad 1 \leqslant i \leqslant 2 k-2$.

Recall now the following statement proven in the Appendix of [Voi02].

Proposition 10. For $q>0, q^{\prime} \geqslant 0, H^{p}\left(G, \mathcal{L}^{-q} \otimes S^{q^{\prime}} \mathcal{E}\right)=0$ if $p \neq k+1,2 k+2$. For $p=k+1$, $H^{p}\left(G, \mathcal{L}^{-q} \otimes S^{q^{\prime}} \mathcal{E}\right)=0$ if $-q+q^{\prime}+1<0$ or $q \leqslant k+1$.

(Note the shift of notation from $k$ in [Voi02] to $(k+1)$ here, which is due to the fact that we are now working with a space $H^{0}(E)$ of rank $k+3$ instead of $k+2$.)

The vanishing (ii) follows directly from this Proposition. The vanishing (iii) follows from the fact that $H^{-1}$ has trivial cohomology along the fibers of $p: P \rightarrow G$. For the vanishing (i), we use the exact sequence on $P$,

$$
0 \rightarrow \mathcal{L} \otimes H^{-1} \rightarrow \mathcal{E} \rightarrow H \rightarrow 0
$$

It provides the exact sequence

$$
0 \rightarrow \mathcal{L} \otimes H^{-1} \otimes S^{k-3} \mathcal{E} \rightarrow S^{k-2} \mathcal{E} \rightarrow H^{k-2} \rightarrow 0 .
$$

Hence we see that (i) is implied by the vanishings

$$
\begin{gathered}
H^{i}\left(P, S^{k-3} \mathcal{E} \otimes \mathcal{L}^{-i}\right)=0, \quad 1 \leqslant i \leqslant 2 k-1, \\
H^{i}\left(P, H^{k-1} \otimes \mathcal{L}^{-i-1}\right)=H^{i}\left(G, S^{k-1} \mathcal{E} \otimes \mathcal{L}^{-i-1}\right)=0, \quad 1 \leqslant i \leqslant 2 k-1,
\end{gathered}
$$

and they are both consequences of Proposition 10.

For the vanishing (iv), one notes that $K_{P / G}$ is equal to $H^{-2} \otimes \mathcal{L}$. Hence we have

$$
\begin{aligned}
H^{i+3}\left(P, S^{k-2} \mathcal{E} \otimes \mathcal{L}^{-i-1} \otimes H^{-2}\right) & =H^{i+2}\left(G, S^{k-2} \mathcal{E} \otimes \mathcal{L}^{-i-1} \otimes R^{1} p_{*} H^{-2}\right) \\
& =H^{i+2}\left(G, S^{k-2} \mathcal{E} \otimes \mathcal{L}^{-i-2}\right)
\end{aligned}
$$

This vanishes for $1 \leqslant i \leqslant 2 k-1$ by Proposition 10 .

To conclude, ( $\mathrm{v})$ is proved as follows. We have as above

$$
\begin{aligned}
H^{i+4}\left(P, S^{k-2} \mathcal{E} \otimes \mathcal{L}^{-i-1} \otimes H^{-3}\right) & =H^{i+3}\left(G, S^{k-2} \mathcal{E} \otimes \mathcal{L}^{-i-1} \otimes R^{1} p_{*} H^{-3}\right) \\
& =H^{i+3}\left(G, S^{k-2} \mathcal{E} \otimes \mathcal{L}^{-i-2} \otimes \mathcal{E}^{*}\right)
\end{aligned}
$$

using relative Serre duality and $R^{0} p_{*} H=\mathcal{E}$ on $G$. Since $\mathcal{E}^{*}=\mathcal{E} \otimes \mathcal{L}^{-1}$, the last term is equal to

$$
H^{i+3}\left(G, S^{k-2} \mathcal{E} \otimes \mathcal{E} \otimes \mathcal{L}^{-i-3}\right) .
$$

By the exact sequence

$$
0 \rightarrow \mathcal{L} \otimes S^{k-3} \mathcal{E} \rightarrow S^{k-2} \mathcal{E} \otimes \mathcal{E} \rightarrow S^{k-1} \mathcal{E} \rightarrow 0
$$




\section{GREEN'S CANONICAL SYZYGY CONJECTURE FOR GENERIC CURVES OF ODD GENUS}

we see that the vanishing $H^{i+3}\left(G, S^{k-2} \mathcal{E} \otimes \mathcal{E} \otimes \mathcal{L}^{-i-3}\right)=0$ for $1 \leqslant i \leqslant 2 k-2$ is a consequence of the vanishings

$$
\begin{aligned}
& H^{i+3}\left(G, S^{k-3} \mathcal{E} \otimes \mathcal{L}^{-i-2}\right)=0, \\
& H^{i+3}\left(G, S^{k-1} \mathcal{E} \otimes \mathcal{L}^{-i-3}\right)=0,
\end{aligned}
$$

for $1 \leqslant i \leqslant 2 k-2$, which both follow from Proposition 10 .

This concludes the proof of (59), and the proof of Lemma 10 will then be concluded with the proof of Lemma 11.

Proof of Lemma 11. Since the map

$$
H^{2 k+1}\left(\widetilde{G}^{\prime}, S^{k-2} \mathcal{E} \otimes H^{-1} \otimes \mathcal{L}^{-2 k}\right) \rightarrow H^{0}(E(-\Delta)) \otimes H^{2 k+1}\left(\widetilde{G}^{\prime}, S^{k-2} \mathcal{E} \otimes \mathcal{L}^{-2 k}\right)
$$

is induced by the inclusion

$$
H^{-1} \subset H^{0}(E(-\Delta)) \otimes \mathcal{O}_{\widetilde{G}^{\prime}},
$$

which is dual to the evaluation map, where $H^{0}(E(-\Delta))^{*}$ is identified to $H^{0}\left(\widetilde{G}^{\prime}, H\right)$, we see that its dual is equal to the multiplication map

$$
H^{0}(E(-\Delta))^{*} \otimes H^{0}\left(\widetilde{G}^{\prime}, K_{\widetilde{G}^{\prime}} \otimes S^{k-2} \mathcal{E}^{*} \otimes \mathcal{L}^{2 k}\right) \rightarrow H^{0}\left(\widetilde{G}^{\prime}, K_{\widetilde{G}^{\prime}} \otimes H \otimes S^{k-2} \mathcal{E}^{*} \otimes \mathcal{L}^{2 k}\right) .
$$

The canonical bundle of $\widetilde{G}^{\prime}$ is equal to $\mathcal{L}^{-k-2}$, because $\widetilde{G}^{\prime}$ is the complete intersection of two members of $|H|$ in $P$ and $K_{P}=\mathcal{L}^{-k-2} \otimes H^{-2}$.

Thus we have to prove that the multiplication map

$$
H^{0}(E(-\Delta))^{*} \otimes H^{0}\left(\widetilde{G}^{\prime}, S^{k-2} \mathcal{E}^{*} \otimes \mathcal{L}^{k-2}\right) \rightarrow H^{0}\left(\widetilde{G}^{\prime}, H \otimes S^{k-2} \mathcal{E}^{*} \otimes \mathcal{L}^{k-2}\right)
$$

is surjective.

Since $\mathcal{E}^{*} \otimes \mathcal{L} \cong \mathcal{E}$, this is equivalent to the surjectivity of the multiplication map

$$
H^{0}(E(-\Delta))^{*} \otimes H^{0}\left(\widetilde{G}^{\prime}, S^{k-2} \mathcal{E}\right) \rightarrow H^{0}\left(\widetilde{G}^{\prime}, H \otimes S^{k-2} \mathcal{E}\right) .
$$

This follows from the surjectivity of the multiplication map

$$
H^{0}(P, H) \otimes H^{0}\left(P, S^{k-2} \mathcal{E}\right) \rightarrow H^{0}\left(P, H \otimes S^{k-2} \mathcal{E}\right),
$$

and of the restriction map

$$
H^{0}\left(P, H \otimes S^{k-2} \mathcal{E}\right) \rightarrow H^{0}\left(\widetilde{G}^{\prime}, H \otimes S^{k-2} \mathcal{E}\right) .
$$

\section{ACKNOWLEDGEMENT}

I am very indebted to the referee for pointing out a mistake in my original proof of Proposition 8 and for helpful comments.

\section{REFERENCES}

Apr04 M. Aprodu, Green-Lazarsfeld's gonality conjecture for a generic curve of odd genus, Int. Math. Res. Notices 63 (2004), 3409-3416.

Apr05 M. Aprodu, Remarks on syzygies of d-gonal curves, Math. Res. Letters 12 (2005), 387-400.

AV03 M. Aprodu and C. Voisin, Green-Lazarsfeld's conjecture for generic curves of large gonality, C. R. Acad. Sci. Paris Sér. I 336 (2003), 335-339.

Ein87 L. Ein, A remark on the syzygies of the generic canonical curve, J. Differential Geom. 26 (1987), $361-365$ 


\section{GREEN'S CANONICAL SYZYGY CONJECTURE FOR GENERIC CURVES OF ODD GENUS}

GL84 M. Green and R. Lazarsfeld, A simple proof of Petri's theorem on canonical curves, in Geometry today, Giornate di Geometria Roma, Progr. Math., vol. 60 (Birkhäuser, Boston, MA, 1984), 129-142.

GL87 M. Green and R. Lazarsfeld, Special divisors on curves on a K3 surface, Invent. Math. 89 (1987), $357-370$.

Gre84 M. Green, Koszul cohomology and the geometry of projective varieties, with an appendix by M. Green and R. Lazarsfeld, J. Differential Geom. 19 (1984), 125-171.

HR98 A. Hirschowitz and S. Ramanan, New evidence for Green's conjecture on syzygies of canonical curves, Ann. Sci. École Norm. Sup. (4) 31 (1998), 141-152.

Laz86 R. Lazarsfeld, Brill-Noether-Petri without degenerations, J. Differential Geom. 23 (1986), 299-307.

Sch86 F.-O. Schreyer, Syzygies of canonical curves and special linear series, Math. Ann. 275 (1986), $105-137$.

Sch91 F.-O. Schreyer, A standard basis approach to the syzygies of canonical curves, J. reine angew. Math. 421 (1991), 83-123.

Tei02 M. Teixidor, Green's conjecture for the generic r-gonal curve of genus $g \geqslant 3 r-7$, Duke Math. J. 111 (2002), 195-222.

Voi02 C. Voisin, Green's generic syzygy conjecture for curves of even genus lying on a K3 surface, J. Eur. Math. Soc. 4 (2002), 363-404.

Voi88 C. Voisin, Courbes tétragonales et cohomologie de Koszul, J. reine angew. Math. 387 (1988), 111-121.

Claire Voisin voisin@math.jussieu.fr

Institut de mathématiques de Jussieu, CNRS, UMR 7586, Paris, France 